\title{
LA IMPORTANCIA DE LOS VALORES EN LA VIDA PERSONAL Y SOCIAL: ENFOQUES Y MEDICIÓN
}

\author{
AURELIO VILLA SÁNCHEZ ${ }^{1}$
}

RESUMEN: Los valores constituyen un tema intemporal, siempre se ha hablado de los valores, y se seguirá hablando, porque es consustancial al ser humano. Se reitera recurrentemente la crisis de valores en las diferentes épocas. Sin embargo, los valores son la guía de actuación de las personas, las instituciones y las sociedades, y es normal que en función de los factores de cambio cultural se modifiquen, se adapten a nuevas perspectivas, pero es muy importante que cualquiera de estos cambios no haga perder el norte. La brújula de los valores debe ser la herramienta que nadie (personas, grupos, instituciones, y sociedad) nunca debe perder. El propósito de este artículo es presentar una vista panorámica de algunos enfoques que existen sobre la temática de los valores y su aplicación en diferentes ámbitos. Los valores desde el punto de vista educativo se convierten en un foco clave en el aprendizaje para muchos modelos educativos desde la "clarificación de valores» hasta la "educación en valores». Los valores en la educación religiosa, que aquí se especifican en la pedagogía jesuitica. Los valores como reflejo de las actitudes y comportamientos de los pueblos que configuran los países en el mundo, y que hoy es posible disponer de este gran espejo gracias a las encuestas mundiales sobre valores. Los valores como centro de las organizaciones y como aspecto nuclear de su funcionamiento institucional. Los valores desde las aportaciones metodológicas desde los estudios pioneros de Milton Rokeach hasta los enfoques más difundidos como S.H. Schwartz. En sintesis, se trata de un intento inacabado pero que abre el obturador para poder captar la luz en un momento determinado.

PALABRAS CLAVE: valores; actitudes; encuesta valores; metodología valores; enseñanza y aprendizaje de valores.

\section{The importance of values in personal and social life: approaches and measurement}

ABSTRACT: Values are a timeless subject: they have always been a field of study and will continue to be, as they are inherent to the human being. The crisis of values is consistently mentioned throughout history. However, values guide the actions of individuals, institutions and societies, and it is normal that, in accordance with cultural change, they should be modified and adapted to new perspectives,

1 Profesor emérito; Universidad de Deusto; Aurelio.villa@deusto.es 
but it is very important that none of these changes makes us lose our way. The compass of values should be the tool that no one (people, groups, institutions, and society) should ever lose. The purpose of this article is to present a panoramic view of some approaches to the subject of values and their application in different areas. Values, from an educational perspective have become a key learning focus for many educational models, ranging from "value clarification" to "values education». Other approaches include values in religious education, which are specified here in Jesuit pedagogy, as well as values reflecting the attitudes and behaviours of the peoples who shape the countries across the world, a mirror of society available by means of global values surveys. Values are at the centre of organizations and form a core aspect of their institutional functioning. Values have been the focus of methodological contributions from Milton Rokeach's pioneering studies to the most widespread approaches like that of S.H. Schwartz. In short, this article is an unfinished attempt to open the shutter and shed some light on the issue of values at this point of time.

KEY WORDS: values; attitudes; survey of values; methodology of values; teaching and learning of values.

«Volver a los valores que propendan el bien común a través de las prácticas educativas acordes con la humanidad tanto del que enseña como del que aprende».

Marco Fabio Quintiliano

\section{INTRODUCCIÓN}

El tema de los valores inunda todas las áreas científicas, sociales y cívicas. Los valores representan el espíritu humano, lo que le mueve en la vida, lo que le permite jerarquizar lo que pretende, lo que desea, lo que lleva a cabo.

Los valores expresan nuestra mirada en el mundo, lo que queremos cambiar, la lucha por la mejora personal, social y de la humanidad. Cada persona y cada institución tiene y muestra sus valores, los que quiere que conozcan los demás, la razón por la que una persona vive, y visiona su futuro a través de los valores que intenta desarrollar.

La práctica de los valores conlleva superar dilemas, elegir entre aspectos que son queridos, a discriminar en razón del valor más alto que cada persona, grupo o institución ha situado en lo más alto de su jerarquía valórica.

El tratamiento de los valores es inabarcable en un artículo como el presente, aquí, fundamentalmente tendremos una mirada desde la perspectiva 
educativa, aunque diferenciando aspectos como modelos de medición de valores, valores desde las instituciones, el tratamiento pedagógico de los valores, y una visión holística de los valores a través de las grandes encuestas a la población mundial.

Como señala Bautista Vallejo (2001):

Dentro de la Psicología, sin duda impregnada de un planteamiento filosófico que le da revestimiento, por lo que no puede desprenderse del todo de los planteamientos anteriores, tampoco hay unidad en las proposiciones. Para autores como Maslow (1964), Rokeach (1968) y Williams (1968), entre otros, los valores han de situarse en la persona; a esto se enfrentan las sugerencias de Campbell (1963) y otros que sitúan a los valores en los objetos. Para Rokeach (1968), autor que ha estudiado en profundidad la relación entre conceptos tan próximos como creencias, actitudes y valores, el valor es un tipo de creencia emplazada en el centro del sistema total de creencias de una persona acerca de cómo se debe o no se debe comportar; o, también, acerca de algún objetivo en la existencia que es digno de esfuerzo o sacrificio en conseguirlo. Los valores son, por ende, ideales o abstractos independientes de cualquier objeto específico o situación concreta de actitud, los cuales representan las creencias de una persona sobre los modelos ideales de conducta y sobre los últimos fines también ideales. (Vallejo, 2001, p. 195)

En sentido genérico, afirman Alarcos y García (2009, p. 144), se pueden definir los valores como cualidades que poseen las cosas en relación con el ser humano. Los valores son, en primer lugar, cualidades pertenecientes a las cosas. Los valores poseen una doble dimensión: objetiva, dado que son cualidades que pertenecen a la realidad y no pueden reducirse a lo que el hombre sienta o estime respecto a ellas, y subjetiva, porque sin relación con el ser humano, sin que éste pueda captar y desvelar su existencia, los valores tampoco tendrían existencia independiente. Por tanto, los valores son aquellas cualidades que hacen ser una realidad dada digna de una de una determinada estimación o consideración axiológica por parte de un sujeto que valora.

Los valores tienen, pues, un significado referencial en función del cual interpretamos y estimamos los diferentes hechos, situaciones o realidades de nuestra existencia.

Los autores citados recogen el origen del término valor, que procede del verbo latino valere, que significa «estar bien, tener salud, vigor, fuerza y energía». Un valor es algo que «está bien», que posee las características propias de lo bueno. Entendemos, por tanto, «el bien» o «lo bueno», al menos en sentido propio, como sinónimos de «valor» o de «valioso». El diccionario de la Real Academia de la Lengua Española, define el valor como «cualidad que poseen algunas realidades, consideradas bienes, por lo cual son estimables».

Los valores no sólo actúan como referentes culturales, sino como vectores decisionales y preferenciales de la persona hacia lo que es valioso en sí 
mismo, tanto para ella como para la sociedad en la que vive. Alarcos Martínez y García Férez (2009), citan a Reiner (1985, p. 19) quién define el valor como: eso que en un ser hace que éste se nos presente como digno y, por tanto, como grato. Para Victoria Camps los valores son: cualidades de las cosas que promueven nuestro interés, que atraen y repelen, y sólo puede tener valor aquello cuya existencia complace.

Existen muy diversas definiciones del término valor, pero una definición sencilla para un término complejo como es el de valores, es la propuesta por Hall y Tonna, como prioridad de actuación o prioridad vital.

Los valores requieren una estructura jerárquica, por eso hablamos en términos de medición de escala de valores. Cada persona puede tener una escala de valores distinta, los que considera más importantes y los que son menos relevantes para esa persona, utilizamos entonces el término valores personales. Esto mismo sucede con las instituciones sociales y empresariales. Una gran parte de las mismas, tienen su declaración de valores en sus documentos de responsabilidad social, o en sus documentos fundacionales. Estos valores se convierten en el marco que inspira sus principios activos, sus procedimientos y sus formas de relacionarse con el mundo.

\section{LOS VALORES DESDE EL PUNTO DE VISTA EDUCATIVO}

Esta publicación quiere rendir homenaje al profesor Pedro Morales Vallejo, S.J., dedicado al estudio, la enseñanza y la investigación, que ha ejercido su profesión con valores de dedicación, entrega, generosidad y solidaridad con los estudiantes más necesitados,

Entre los diversos y múltiples temas de estudios e investigaciones realizados por el autor, como atestigua su amplia publicación, quiero subrayar el tema de los valores y actitudes en los centros educativos.

Ya en el año 1985, llevó a cabo junto con el P. López Yarto un estudio empírico sobre los valores de centros jesuíticos de España. Utiliza en este estudio una metodología en el que los participantes tienen que poner en orden de preferencia 12 valores.

Dos aspectos a resaltar de este estudio: por un lado, la aportación de una metodología para evaluar los valores en un aula o en pequeños grupos de forma sencilla y práctica a través del estudio de rangos; y por otro un estudio empírico interesante con una muestra de más de 3.000 estudiantes para conocer los valores de estudiantes de centros educativos religiosos y públicos.

Como puede observarse de la mera comparación de los datos, los valores constantemente clasificados en primeros lugares son paz, amor y la bina 
libertad-igualdad (aunque éstos cambian el orden en los colegios no jesuíticos). Como era de esperar se encuentra una mayor diferencia de orden que ocupa el valor sentido religioso, mucho más elevado en los colegios de la Compañía.

Diez años más tarde, Morales (1995) llevó a cabo un estudio para examinar el influjo docente sobre las actitudes y valores de los estudiantes. Parte de una definición sencilla de valor: un valor es aquello que consideramos importante, y más importante que otras cosas. Y como es importante se convierte en criterio en la toma de decisiones institucionales y personales.

El autor parte de tres consideraciones importantes:

1. Las universidades tienen objetivos explícitos en el campo de los valores.

2. El que por parte de la universidad no se pretenda influir no quiere decir que no se influya de hecho, $y$ de muchas maneras; $y$ puede ser que esos influjos no sean precisamente los deseados.

3. Por qué puede haber resistencias a hacer explícitas las cuestiones de valores.

Sobre el primer punto Morales señala algunas universidades que declaran los valores definidos en sus documentos institucionales. Actualmente es muy difícil encontrar una universidad que no haga público en su web la misión, la visión y los valores que define como orientación de su quehacer académico. Estas declaraciones son tan generales, que si no existen algunos procedimientos más operativos para trabajarlos se reducen a meras y bonitas declaraciones.

Respecto al segundo punto, afirma, aunque al profesor no siempre le guste la idea de influir en los valores de los alumnos, no por eso deja de ejercer un determinado influjo, y en este contexto es conveniente recordar que los resultados conseguidos realmente pueden no corresponder con los resultados pretendidos, v precisamente en el área de los valores y actitudes. Analiza esta situación a través de una propuesta de Yamamoto (1969) en la que se diferencia si el profesor enseña intencionadamente o no, y si el estudiante aprende intencionadamente o no.

Finalmente, sobre el tercer punto, Morales plantea tres posibles razones por las que existen tres tipos de dificultades que surgen a la hora de plantear en el aula cuestiones sobre valores: la propia resistencia de los profesores, las expectativas de los alumnos que van por otros caminos y el no caer en la cuenta de los cómos o no pensar suficientemente en los modos de plantear cuestiones de valores.

En su estudio examina distintos estudios para analizar posibles factores que puedan ser decisivos en el influjo de los estudiantes. Morales (2006) 
dedica a analizar muy profundamente la propuesta de Yamamoto, citado anteriormente, con el propósito de determinar las implicaciones docentes en una enseñanza centrada en el estudiante, y en dónde de modo detallado estudia diversas competencias docentes: diseño de tareas de aprendizaje, evaluación orientada a desarrollar un aprendizaje profundo del estudiante, las competencias interpersonales, y examinar tanto los aprendizaje negativos no pretendidos como los positivos en el ámbito de los valores.

\subsection{LA CLARIFICACIÓN DE VALORES}

En la década de los años 70 se desarrolló una metodología para desarrollar los valores en los estudiantes denominada Clarificación de Valores. La intención de esta metodología no era promover determinados valores, sino ayudar a los estudiantes a reconocer sus propios valores y a que le dieran importancia de vivir con valores.

Nuestros valores son todo aquello por lo que estamos a favor o en contra. Los valores dan sentido y dirección a nuestras vidas. Si nuestros valores son claros, consistentes y bien escogidos, tendemos a vivir de manera significativa y satisfactoria. Si carecemos de valores o son confusos y contradictorios, la vida nos resultará problemática y frustrante. (Howe y Howe, 1977, p. 19)

La clarificación de valores muestra un respeto absoluto por los valores de cada persona, y su propósito es enseñar a los estudiantes a clarificarlos, vivir según esos valores y enseñar a respetar los valores de los demás. No se trata de enseñar valores correctos o incorrectos, sino ayudar a los estudiantes a comportarse y actuar en los diferentes ámbitos de su vida de acuerdo con sus valores.

Las pautas que debe seguir el profesorado para enseñar esta metodología son lograr que los estudiantes puedan:

- escoger libremente sus valores;

- escoger sus valores de entre distintas alternativas;

- escoger sus valores después de sopesar las consecuencias de cada alternativa;

- apreciar y estimar sus valores;

- compartir y afirmar públicamente sus valores;

- actuar de acuerdo con sus propios valores;

- actuar de acuerdo con sus propios valores de manera repetida y constante.

En su obra, Howe y Howe (1977) presentan en realidad un manual didáctico repleto de actividades, ejercicios y prácticas para que el profesorado 
tenga suficientes herramientas para desarrollar esta metodología, así como cuestionarios para hacer reflexionar a los estudiantes sobre un panorama de temas y ámbitos de la vida personal y social.

\subsection{LA EDUCACIÓN EN VALORES}

Como afirma Gil Martínez (1998), los valores juegan un papel central en el dinamismo de la personalidad como metas de autorrealización personal, como ideales que regulan los comportamientos individuales o colectivos, como marcos de conocimiento desde los que nos percibimos a nosotros mismos y a los demás, o como análisis de las situaciones en las que vivimos para decirnos por un tipo de acción o por otro. Las instituciones educativas, especialmente la escuela, deben plantearse, en una época de confusión valórica, ayudar a los jóvenes a identificar y clarificar sus propios valores para que tomen decisiones auténticamente suyas. Consideramos que éste es el gran reto de las escuelas de nuestro tiempo.

Un paso importante es que ayudar a los estudiantes a diagnosticar sus propios valores, reconocerlos es el primer paso para actuar en consonancia y realmente que sean la guía del comportamiento. Gil Martínez propone dos metodologías de carácter complementario para formar actitudes y valores en la escuela. Estas dos metodologías son:

a) Construir los valores de forma interdisciplinar, es decir, a través de todas las estructuras del currículum, como un tema transversal que afecte a una o a diferentes disciplinas, las cuales se verían necesariamente coordinadas por sus actividades a favor de los valores elegidos.

b) Asignar a la enseñanza de valores y actitudes un lugar y un tiempo específicos dentro del proceso educativo, como son las horas de tutoría lectiva.

Y señala diez valores a trabajar en la educación: dignidad de la persona, tolerancia, libertad, autoestima, conciencia crítica, justicia, respeto al medio ambiente, solidaridad, diálogo y amor-amistad.

Muchos de estos diez valores coinciden también con las propuestas de los listados de universidades (solidaridad, justicia, dignidad, libertad, respeto al medio ambiente, aparecen reiteradamente).

Oyola (2012) expresa la importancia de construir una sociedad con valores sólidos y profundos es incuestionable. La autora recoge la definición de Touriñán (2006): «los valores como criterios que rigen el comportamiento de los seres humanos, identifican a un individuo con la manera de estar en el mundo del grupo al que pertenecen afirmando así una determinada concepción de la humanidad» (p. 3). 
La autora propone un modelo de aprendizaje-servicio como estrategia para desarrollar valores. En la idea de Furco (2000) quién lo menciona en primer lugar, los proyectos de aprendizaje servicio se coordinan en las instituciones para brindar servicio a las comunidades necesitadas. Esto quiere decir, que el proyecto no se realiza en el salón de clases. El estudiante se matrícula en el curso y luego hace una investigación de las necesidades que tiene su comunidad. Posteriormente se integra como un recurso activo en la agencia o institución seleccionada poniendo en ejecución los conocimientos adquiridos a lo largo de su experiencia universitaria. En segundo lugar, es a través del aprendizaje servicio que el estudiante fortalece su experiencia universitaria (Oyola, 2012, p. 26).

La estrategia de aprendizaje-servicio nació en Estados Unidos y actualmente existen numerosas asociaciones y universidades que la desarrollan como un arte relevante de su acción social y de formación en valores por parte de sus estudiantes.

Pueden verse distintas aplicaciones de proyectos de aprendizaje-servicio (Villa et al. (2013) en el que se exponen ejemplos de proyectos en 14 universidades, Santos, Sotelino y Lorenzo (2015), García (2018), García, Mugarra y Villa (2016) y Aramburuzabala, Cerrillo, y Tello, (2015); Opazo, Aramburuzabala, y McIlrath (2019).

El aprendizaje-servicio es una metodología muy apta para el desarrollo de los valores sociales junto con el voluntariado y para el desarrollo de otras actitudes como la responsabilidad, la actitud cívica, la prestación de ayuda desinteresada, y el poner el foco en los demás, además de poner en práctica las competencias académicas de cada profesión (medicina, derecho, educación, ingeniería, etc.), ayudan a grupos o barrios con escasos medios en esas áreas dónde llevan a cabo iniciativas y proyectos basados en sus necesidades detectadas.

\subsection{LA CONSTRUCCIÓN DE LOS VALORES: ES UNA CUESTIÓN ÚNICAMENTE PERSONAL O TAMBIÉN SOCIAL}

El hombre virtuoso, según Aristóteles, es el hombre libre que conforma su vida a unas actitudes, hábitos y maneras de ser que acabarán constituyendo su personalidad. Llegará un momento en que la tolerancia, la convivencia, la participación política o las buenas maneras no serán una obligación, sino una costumbre. Ese es el sentido del ethos moral: la construcción y formación de la propia naturaleza - una naturaleza que, como decían los renacentistas, puede llegar a ser muchas cosas-, de acuerdo con unas directrices o con una idea que es el horizonte de la ciudadanía. (Camps, 1998, p.16) 
Es evidente que los valores tienen que constituirse de forma personal, pero eso no debe significar hacerlo de modo independiente de la sociedad en la que uno vive y convive. Camps defiende el valor del civismo, y ello significa no hablar sólo de formas sino de formarse un criterio: un criterio individual, pero no a espaldas de la sociedad.

Una objeción a la idea de ciudadano como mero sujeto de derechos tiene que ver también con el civismo. La ilustración nos dejó el mensaje del universalismo: hay que perseguir aquellos valores y principios que puedan ser universales. Hay que procurar que los derechos lo sean para todos sin exclusión de nadie. Camps trae a colación una frase de Marion Young (1990) que es importante resaltar: «todos los hombres nacen libres e iguales» decía el principio máximo de la ley natural. Pero las mujeres, los no propietarios, los esclavos, quedaban fuera de ese «todos». Y para corregir ese engaño no basta repetir fórmulas de igualdad. Ni siquiera basta procurar no excluir a nadie sin más. Hacen falta políticas diferenciadas. Hace falta «tratar diferente a los diferentes».

Cada día es mayor el número de personas conscientes de que la crisis económica y financiera global tiene también que ver con valores y normas éticas comunes... Sin moral, las leyes no pueden subsistir, y ninguna disposición legal se puede llevar a efecto en ausencia de una conciencia moral basada en ciertos principios éticos elementales. Pero esto ¿es solo una cuestión de moral individual? De ningún modo: es también una cuestión de moral corporativa, y afecta al conjunto de la economía global de mercado. De ahí su primera reflexión: el fallo de los mercados, de las instituciones y de la moral exige un marco ético. (Hans Küng, 2012, p. 29)

Küng (2012, p. 34) aboga por un manifiesto por una ética global sustentada en dos principios básicos subyacentes a todos los valores y normas éticas: la humanidad y la reciprocidad. Y señala cuatro valores esenciales:

- un compromiso con la cultura de la no violencia y el respeto por la vida,

- un compromiso con la cultura de la equidad y un orden económico justo,

- un compromiso con la cultura de la veracidad y la tolerancia, y

- un compromiso con la cultura de la colaboración y la igualdad de derechos entre hombres y mujeres.

La necesidad de una formación en valores en el ámbito educativo desde los niveles de primaria hasta el universitario es patente. Es verdad que la gran mayoría de las universidades tienen explícitos en sus documentos programáticos los valores que guían su actividad y constituyen el marco fundamental de su misión. Cualquiera puede entrar en la web de una universidad 
y encontrar los valores que sustentan la formación de sus actividades académicas. Normalmente, el número de valores que definen estos marcos suelen estar entre ocho y doce valores como promedio, sin embargo, la clave es cómo desarrollarlos, y para tener incidencia, es necesario compartir los valores y trabajarlos en equipo.

\subsection{LOS VALORES DESDE UNA PERSPECTIVA ESPIRITUAL Y RELIGIOSA}

Los valores constituyen el elemento central de las religiones y, por ende, de las órdenes religiosas que se dedican al ámbito educativo. Los valores son considerados la guía y referencia clave para el comportamiento de la personas e instituciones.

Una versión más lúdica puede verse en el estudio de Lanuza-Avello, Cabezuelo-Lorenzo y García-Plazas Vegas (2016) se centran en el análisis de la producción audiovisual The Walking Dead para comprobar cómo esta serie de ficción utiliza distintos recursos temáticos y audiovisuales para plantear ideas que podemos relacionar con la cosmovisión y la moral cristianas, y del enfoque humanista. A través de sus personajes y de las situaciones que van enfrentándose puede verse el comportamiento de los valores humanistas que van haciéndose patentes en el desarrollo de la historia.

Los diversos fundadores de las órdenes religiosas dedicadas a la educación plantearon desde su inicio los valores que deseaban promover a través de sus actividades y tareas educativas. Lógicamente el propósito de estas órdenes religiosas era promover los valores desde la óptica católica.

\section{LA PERSPECTIVA JESUÍTICA DE LOS VALORES}

Al describir los valores de «nuestra educación» (referidos a la educación jesuítica), el Padre Kolvenbach (1989) afirma:

[...] valor significa literalmente algo que tiene un precio, que es querido, que es de mucha estima o que vale la pena; consiguientemente, algo por lo que uno está dispuesto a sufrir o a sacrificarse, algo que es una razón para vivir y, si fuera preciso, para morir. Así, los valores aportan a la vida la dimensión del «significar algo para alguien». Son los raíles que mantienen al tren en su camino y facilitan el deslizarse suavemente, con rapidez y determinación. Los valores proporcionan motivos. Dan identidad a la persona, le ponen facciones. Nombre, carácter. Sin valores uno fluctuaría como los troncos en los remolinos del Potomac. Los 
valores son algo que ocupa el centro de la propia vida, marcando su extensión y su profundidad. (Kolvenbach, p. 60)

El tema de los valores es un tema recurrente en todos sus discursos dirigidos a las diferentes universidades e instituciones de educación jesuíticas. En Frascati (Roma) Kolvenbach (1985) expresaba: «Ignacio sabía perfectamente que un colegio es un colegio y una universidad, una Universidad. Tienen su propia finalidad y no son meras oportunidades para la evangelización o la defensa de la fe. Puesto que la difusión de la Reforma se debía en parte a la decadencia de los estudios, Ignacio -en post del hombre integral, "virtuosos y doctos» (Const. 308)- adoptó los valores y la transformación de los valores que un colegio o una universidad pueden garantizar y desarrollar» (p. 37). Kolvenbach deja claro el mensaje que no se trata de convertir a los centros y universidades en una feligresía sino en la formación integral de los estudiantes y buscar el sentido último de la vida, y poner el foco en el servicio a los demás.

En Georgetown afirmaba: Los valores tienen tres puntos de anclaje. En primer lugar, están anclados en la «cabeza». Yo percibo, veo las razones por las que algo tiene valor y estoy intelectualmente convencido de los que la cosa vale. Los valores también están anclados en el «corazón». No sólo la lógica de la cabeza, sino también el lenguaje del corazón me dice que algo es valioso, de tal forma que no sólo soy capaz de percibir algo como valioso, sino que también quedo afectado por el valor que representa. «Donde está tu tesoro, allí está también tu corazón». Cuando la cabeza y el corazón están interesados, la persona está interesada. Esto nos lleva al tercer punto de anclaje, dicho en una palabra: «la mano». Los valores conducen, y ello de forma necesaria, a decisiones y acciones. «El amor se muestra con obras, no con palabras» (p. 60).

Los valores, por tanto, nacen y se desarrollan desde la persona completa, y se manifiestan en las opciones de vida y en la elección de cada conducta consecuente en cada situación de la vida. Los valores representan los grandes indicadores del pensamiento y el comportamiento humano.

En el segundo centenario de la enseñanza jesuítica en los Estados Unidos el P. Kolvenbach (1989) define el objetivo basado en los valores del tipo de educación deseado, afirmando: formar hombres y mujeres para los demás- no se realizará a no ser que haciendo que ese objetivo inspire todos nuestros programas de enseñanza, desafiemos a nuestros alumnos para que reflexionen sobre los valores que subyacen en lo que estudian. Bien a nuestro pesar hemos aprendido que adquirir conocimientos no humaniza forzosamente (p. 82). En este sentido, señalaba en México (en la Universidad Iberoamericana) cuando decía: 
[...] el solo adueñarse de conocimientos no humaniza automáticamente. Una actitud crítica de la cultura, a la que me he referido, sólo es factible si descubrimos los caminos que hagan posible a los estudiantes el formar hábitos de reflexión para fijar valores y para que capten las consecuencias que, para la nación y para la humanidad, tienen lo que estudian. Los hábitos se desarrollan únicamente con una práctica continua y planeada. Por esto, la meta de formar hábitos de reflexión necesita ser trabajada por todo el profesorado de la institución educativa jesuítica y en todos los contenidos académicos (p. 95).

Kolvenbach (1991) muestra su preocupación por una enseñanza meramente basada en el desarrollo cognitivo, y defiende la formación integral y su preocupación por una enseñanza ética cuando advierte con la siguiente reflexión: «la universidad necesita reforzar su preocupación por los problemas éticos y por los aspectos éticos de todos los problemas de que se ocupa; lo que, aunque lo incluye no significa algo más que yuxtaponer a los programas una disciplina de la correspondiente deontología. Significa descubrir e iluminar la relación que los diversos conocimientos teóricos y prácticos tienen en la persona humana, y consiguientemente, modificar profundamente la visión global y la orientación de cada disciplina» (p. 110).

En su alocución en UNISINOS (Brasil), Kolvenbach (1992) reflexionaba sobre el cambio de cultura y la crisis producida en la sociedad brasileña: entre la aceptación resignada del predominio creciente de una cultura importada, como la moderna, ella misma en crisis y en desintegración, y la pretensión irreal de reafirmar la cultura tradicional en sus varias formas, se abre una tercera vía. La crisis de los valores que agita a la sociedad brasileña, el vacío ético provocado por la caída de los sistemas anteriormente vigentes, -e inherentes, a su vez, a la mentalidad emergente-, proporcionan la oportunidad de la creación de un nuevo «ethos» social, adecuado a la índole del pueblo de este país. De hecho, se siente por todas partes la aspiración a un proyecto cultural, que fundamente la convivencia de todos los ciudadanos (p. 121).

Aunque el P. Kolvenbach (2001) disertó en todas sus intervenciones en las universidades jesuíticas sobre los valores, recogemos aquí a modo de cierre una reflexión realizada en Monte Cucco (Roma):

No nos hagamos ilusiones: el conocimiento no es neutro, porque implica siempre valores y una determinada concepción del ser humano. La docencia y la investigación no pueden dar la espalda a la sociedad que las rodea. La manera como la primera Compañía entró en interacción con el mundo de la cultura fue precisamente a través de los colegios. La universidad debe ser el lugar donde se airean cuestiones fundamentales que tocan a la persona y a la comunidad humana, en el plano de la economía, la política, la cultura, la ciencia, la teología, la búsqueda de sentido. La universidad debe ser portadora de valores humanos y 
éticos, debe ser conciencia crítica de la sociedad, debe iluminar con su reflexión a quienes se enfrentan la problemática de la sociedad moderna o postmoderna, debe ser el crisol donde se debatan con profundidad las diversas tendencias del pensamiento humano y se propongan soluciones (p. 200).

La competencia de la reflexión y el discernimiento personal son realmente dos características clave de la pedagogía jesuítica. El mero conocimiento sin reflexión e interiorización pierde su significado más preciso del aprendizaje que consiste en la transformación de la persona. Y el centro de la pedagogía jesuítica se resume en el lema: formar hombres y mujeres para los demás.

Como ejemplo de estos valores puede señalarse el marco de valores creado por el Padre Ledesma que ha sido retomado por el que fue General de la Compañía de Jesús, P. Peter Hans Kolvenbach y que se configura por cuatro dimensiones: Utilitas, Iustitia, Humanitas y Fides y que puede verse en Agúndez (2008 a).

El vicerrectorado de Innovación y Calidad de la Universidad de Deusto a través de una comisión ad hoc realizó la tarea de seleccionar y redefinir los valores teniendo en cuenta cada una de las cuatro dimensiones del paradigma Ledesma-Kolvenbach, como puede apreciarse en la Figura 1, que posteriormente fueron ratificados por el Consejo de Gobierno de la Universidad de Deusto y el Rectorado en el Plan Estratégico de 2010.

(A) UTILITAS - Dimensión práctica: la Universidad se propone formar para la excelencia profesional a través de la excelencia académica, asentando a ambas en los valores morales y en la dinámica del magis. (1) Orientación al conocimiento: Disposición a través del aprendizaje abierto al saber y a la investigación, de una adecuada comprensión de la realidad sustentada en la verdad. (2) Innovación y creatividad: Generación de ideas, elaboración y expresión de nuevas propuestas o adecuaciones de propuestas existentes a nuevos contextos, teniendo en cuenta su viabilidad, convirtiéndolas en realidades prácticas y socialmente valiosas. (3) Visión Global: Percepción de una imagen de la realidad sistémica, universal y holística -más allá de la parcialidad, el particularismo y el individualismo-, inspirada en una valoración moral, y ser capaz de comunicarla.

(B) IUSTITIA - Dimensión social: persigue formar personas «para los demás y con los demás», comprometidas, en cuanto ciudadanos, con la construcción de estructuras sociales, económicas y políticas justas, desde el dinamismo de la «opción preferencial por los pobres». (4) Igualdad: Defensa coherente de que todos los seres humanos tienen todos los derechos inherentes a su dignidad -y sus correspondientes deberes-, al tiempo que se reconocen sus diferencias y especificidades. (5) Cultura para la Justicia: Actuación para abordar, confrontar y corregir condiciones de desigualdad y 
opresión humana, lo que supone una redistribución de los recursos, con el fin de hacer efectivo el hecho de que todo ser humano tiene el mismo valor. (6) Compromiso social: Utilización de las propias cualidades y destrezas para realizar una contribución positiva a la sociedad, en especial afrontando las desigualdades, opresiones y marginaciones, a través de la participación cívica, el trabajo profesional y el voluntariado.

(C) HUMANITAS - Dimensión humanista: se propone estimular la formación de la «persona integral», sustentada moralmente en la centralidad de nuestra común dignidad. Por lo que respecta a la vertiente intelectual, que no debe ser exclusiva, fomenta el cultivo de la racionalidad en su complejidad, la que, en el respeto a su autonomía, no se cierra a la fe religiosa. (7) Desarrollo Personal: Cultivo y manifestación de la totalidad integrada de las capacidades en uno mismo y en los demás. (8) Dignidad Humana: Reconocimiento y respeto en la práctica que todo ser humano es en sí mismo valioso, no instrumentalizable, capaz de iniciativa autónoma; lo que le da derecho a desarrollar satisfactoriamente sus capacidades, de manera que puede desarrollar plenamente su potencial. (9) Responsabilidad ética: Actuación de acuerdo con principios morales fundados, discerniendo prudencialmente su aplicación a la realidad, de modo tal que, cumpliendo las exigencias de la justicia que incluye deberes ante los demás y ante sí mismo, se asuman las consecuencias previsibles de las decisiones tomadas.

(D) FIDES - Dimensión religiosa: invita a los estudiantes a procesos de búsqueda del sentido último de la vida. Siendo empáticamente sensible a la pluralidad ante lo religioso, ofrece en concreto el horizonte fundante e integrador de la fe cristiana, el que se expresa en el encuentro personal con el Jesús del evangelio. (10) Búsqueda de sentido: Apertura al descubrimiento de un significado profundo de la propia existencia y de la realidad en su conjunto. (11) Diálogo interreligioso: Orientación a las propias convicciones a la intercomunicación con personas o grupos de otras creencias religiosas desde el respeto y en condiciones de equidad. (12) Fe Cristiana: Apertura experiencial al Misterio del Dios revelado en Jesucristo, que estimula al seguimiento de su persona (Villa y Lemke, 2016, p. 5).

Para un mayor conocimiento de las cuatro dimensiones véase el documento de UNIJES denominado: "Reflexiones $i+m$ ante los nuevos desafíos universitarios» (Documento-Marco, 2007) y el artículo de Villa y Lemke (2016) «El Paradigma Ledesma-Kolvenbach: Origen y concreción en el ámbito universitario.» En estos documentos se intenta operacionalizar estas cuatro dimensiones a través de estrategias y procedimientos para su trabajo académico en las universidades. 
Figura 1. Dimensiones y Planos del PaRadigma Ledesma-Kolvenbach

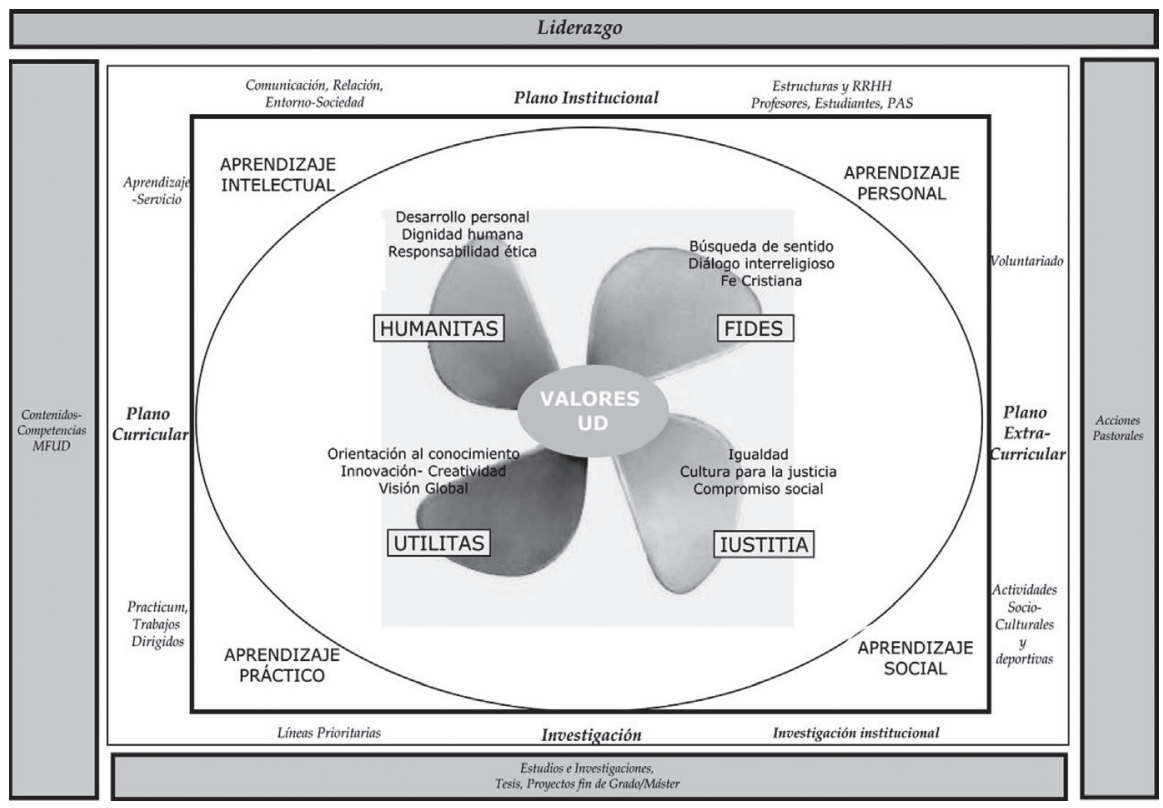

Fuente: reproducción Villa y Lemke (2016, p. 7).

\section{EL ESPEJO DE LOS VALORES EN EL MUNDO}

El reflejo en el espejo de los valores en el mundo puede percibirse en los resultados de la medición de valores a través de las encuestas mundiales.

En 1981, hace casi 30 años se creó la Encuesta Europea de Valores (European Values Surveys) de la que se viene aplicando con regularidad, y hoy puede verse la evolución de los valores en un panorama internacional. Esta encuesta empezó con 24 países y actualmente ya se aplica casi en 100, lo que permite obtener una foto muy actualizada y precisa de los valores de las diferentes sociedades y sus semejanzas y diferencias. Como señalaba el propio Inglehart (2005, p. 22) «lo fascinante es comprobar que los cambios se producen en las motivaciones de las personas, en sus valores fundamentales en sus creencias».

Nuestro amigo y colega José Ignacio Ruiz Olabuénaga, siempre adelantado a su tiempo, editó una publicación en 1984, sobre los estilos de vida con 
autores de primera fila mundial. Entre ellos, estaba Ronald Inglehart quién presentó su enfoque del «Postmaterialismo en un Entorno de Inseguridad». El autor aplicó por primera vez una batería compuesta por los siguientes 12 ítems en la Comunidad Europea y en EE.UU.

- Mantener el orden en la nación.

- Dar a la gente más voz en las decisiones del gobierno.

- Luchar contra la subida de precios.

- Proteger la libertad de expresión.

- Mantener el nivel alto de crecimiento económico.

- Asegurarse de que este país tiene unas fuerzas armadas poderosas.

- Dar a la gente más voz en la decisión de las cosas.

- Tratar de conseguir unas ciudades y paisajes más bonitos.

- Mantener una economía estable.

- Luchar contra el crimen.

- Caminar hacia una sociedad más amistosa y menos impersonal.

- Caminar hacia una sociedad donde las ideas cuenten más que el dinero.

Los ítems en cursiva fueron diseñados para cubrir el énfasis en las metas materialistas; el resto de los ítems fueron diseñados para cubrir las metas post-materialistas. Los resultados confirman que aquellas personas que dan prioridad a una meta materialista tienden a dar máxima a las otras metas materialistas. Por otro lado, también los ítems post-materialistas tienen a ir juntos.

Cada uno de estos dos enfoques influye decisivamente en toda una serie de valores como: los derechos de la mujer, actitudes hacia la pobreza, y otros muchos temas.

La World Values Survey Association anuncia la primera versión del último conjunto de datos de la Encuesta mundial de valores (WVS-7, V1.0). El trabajo de campo para esta séptima ola se llevó a cabo desde mediados de 2017 hasta principios de 2020.

El conjunto de datos WVS-7 incluye más de 300 indicadores, basados en el cuestionario común WVS-7 que está estandarizado en todos los países. También se incluyen variables a nivel macro para cada nación, como la región mundial, el PIB per cápita y el nivel de democracia. El cuestionario WVS-7 cubre 14 temas:

Actitudes y estereotipos de valores sociales (45 elementos); Bienestar social (11 elementos); Capital social, fideicomiso y membresía organizacional (49 puntos); Valores económicos (6 partidas); Corrupción (9 elementos); Migración (10 elementos); post-materialista (6 elementos); Ciencia y 
tecnología (6 artículos); Valores religiosos (12 elementos); Seguridad (21 artículos); Valores y normas éticas (23 elementos); Interés político y participación política (36 puntos);Cultura política y regímenes políticos (25 puntos); Demografía (31 elementos).

Cualquier interesado puede acceder a la base de datos que está de libre acceso, y como puede advertirse la amplitud de temas tratados es muy grande.

Inglehart y Welzel (2013) establecen dos grandes dimensiones del cambio: un giro hacia la modernización en la cultura a partir de una autoridad tradicional hacia una autoridad legal racional, y desde una economía basada en la estabilidad hacia una sociedad en lo que lo predominante es el crecimiento económico.

Desde la perspectiva de los valores de una sociedad tradicional existe un conjunto de características que la configuran: la importancia de la religión, la enseñanza de los niños basada en normas y en la obediencia, el patriotismo, el sentimiento de orgullo nacional. Otros elementos rechazados en las sociedades tradicionales son el aborto, el divorcio. En las sociedades seculares-racionales se aceptan mucho más y en cambio la autoridad es un bien despreciado.

Hace sólo cuatro décadas nos parecería realmente increíble poder tener un mapa mundial de los valores de países con culturas tan diversas, con distintos idiomas y con religiones tan heterogéneas.

Para entender este complejo mapa, Inglehart y Welzel destacan dos dimensiones principales de la variación cultural cruzada en el mundo:

1. Los valores tradicionales versus los valores seculares-racionales.

2. Los valores de supervivencia en comparación con los valores de la autoexpresión.

Los valores tradicionales ponen el énfasis en la religión, las relaciones paterno-familiares, la familia y la tradición.

Los valores secular-racionales son los opuestos a los valores tradicionales, se encuentran por tanto en el polo opuesto del mapa. Y son más partidarios o aceptan mejor el divorcio, el aborto, la eutanasia y el suicidio.

Los valores de supervivencia ponen el foco en la seguridad económica y física. Están relacionados con una relativa perspectiva etnocéntrica y bajos niveles de confianza y tolerancia.

Finalmente, los valores de autoexpresión dan alta prioridad a la protección del medio ambiente, la creciente tolerancia de los extranjeros, la homosexualidad, y la igualdad de género, y la creciente demanda de participación en la toma de decisiones en la vida económica y política. 
Figura 2. Mapa de valores Según la encuesta World Values SurveY

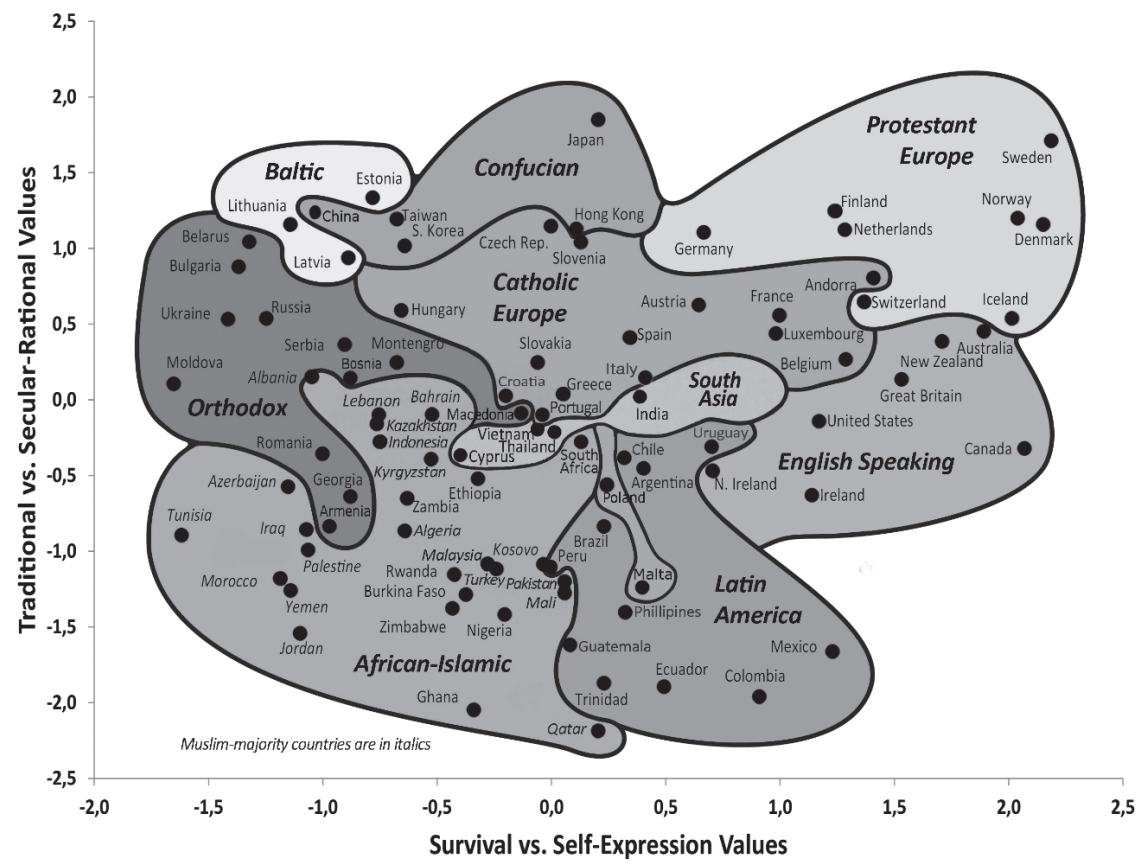

Fuente: De World Values Survey https://commons. wikimedia.org/w/index.php?curid=82456687

Esta visión espacio-valórica del mundo permite de un modo global percibir las agrupaciones de los países en este mapa, configurado por dos ejes: el eje vertical corresponde a los valores tradicionales versus valores secularesracionales y el eje horizontal representa los valores de supervivencia versus autoexpresión.

Los valores de autoexpresión situados en la parte superior derecha del mapa, están situados los países con orientación protestante en Europa (son los países nórdicos: Suecia, Noruega, Finlandia, Dinamarca, y otros países centro europeos como Alemania, Holanda, Suiza. Y también se incluye a Islandia, aunque geográficamente no se sitúe en este espacio físico, pero mantiene valores similares.

En la parte inferior izquierda del mapa, se encuentran los países más tradicionales, muchos de ellos con una orientación religiosa islámica, como una gran parte de países africanos. 
Una gran ventaja de esta encuesta es que se lleva a cabo de forma sistemática y nos permite conocer los valores comparativamente en los diferentes países situados en todos los continentes.

Lo que se puede observar de esta encuesta de valores que se lleva a cabo en casi 100 países es la extracción de conclusiones muy interesantes y que hacen pensar el impacto de los valores en muchos aspectos vitales. Por ejemplo, Inglehart (2005) destacaba una serie de aspectos concluyentes de esta encuesta.

- El desarrollo económico hace descender el índice de natalidad, es en tanto que aporta ciertos cambios culturales, cambios en las expectativas de las personas con respecto a sus vidas, en su forma de ver el mundo.

- La tolerancia es muy importante para la democracia: tolerancia con respecto a los grupos de diferenciales de gais y lesbianas, por ejemplo, que incide en la aceptación de la igualdad de género, etc.

- Se ha dado un proceso de secularización, pero sólo en parte, pues también es verdad, aunque pueda parecer paradójico, que hay hoy en el mundo más personas que nunca con creencias religiosas tradicionales.

- La religión sigue constituyendo un factor vertebrador de vital importancia para la gran mayoría de la población mundial. Aunque es cierto que su influencia decrece en Europa como lo ha hecho también en España, pero continúa siendo muy importante en la mayor parte del mundo.

- Otro elemento relacionado empíricamente con todo esto es el patriotismo, el sentimiento de orgullo por la propia nación.

Según este autor la situación es que tenemos dos dimensiones del cambio, que podríamos resumir de forma esquematizada: un giro hacia la modernización en la cultura a partir de una autoridad tradicional hacia una autoridad legal racional, y desde una economía basada en la estabilidad hacia una sociedad en que lo que predomina es el crecimiento económico. Se trata de un cambio de dirección y de un cambio cultural, algo que podría llamarse postmodernismo, sociedad postindustrial, sociedad del conocimiento.

Se podría ubicar las sociedades del mundo en un mapa bidimensional que tomara en consideraciones estas dos dimensiones que recogiera un gran número de aspectos tales como la religión, la política, la inversión dedicada al ocio y las normas sexuales, familiares y sociales en general. Y estas dos dimensiones, dispuestas de forma estratégica, explican el $75 \%$ de la varianza, incluso un poco más, de entre la multitud de variables en un mundo que utiliza las encuestas. 


\section{LAS ENCUESTAS SOBRE VALORES: MEDICIÓN EN ÁMBITOS ESPECÍFICOS}

Son muchas y variadas las encuestas que se llevan a cabo en distintas poblaciones y diversos sectores, como pondremos algunos ejemplos.

\subsection{VALORES EN LA JUVENTUD Y DE LA POBLACIÓN ADULTA}

En España, la Fundación Santa María (SM) ha venido publicando una encuesta de modo sistemático para conocer los valores de la juventud. Como lo ha hecho en periodos sucesivos, se puede extraer los cambios ocurridos en los valores en los jóvenes en distintos momentos. Los equipos de investigación fueron dirigidos por Elzo $(1994,2006)$ y en otras ocasiones por González (2006).

Elzo también fue el primer investigador que participó en la Encuesta Europea de Valores y publicó los valores de los vascos en tres épocas distintas (1996, 2002).

Por tanto, actualmente se puede conocer los valores de la juventud española y su diferencia según décadas.

\subsection{VALORES POLÍTICOS E INSTITUCIONALES}

Otra encuesta relevante es la que promueve el BBVA. El Estudio Europeo de Valores 2019 de la Fundación BBVA examina un amplio conjunto de valores y actitudes de la población adulta de 5 países europeos: Alemania, Reino Unido, Francia, Italia y España. Los valores y actitudes considerados abarcan los ámbitos público (política, economía, medios, confianza) y privado (religión, ética, ciencia, medio ambiente).

Desde la perspectiva de la esfera pública su presentan los datos de España en comparación de los otros países, señalamos únicamente los grandes epígrafes:

- Interés, información y participación en la esfera pública.

- Autoidentificación ideológica, democracia, papel del Estado e identificación territorial.

- Confianza en grupos e instituciones.

- Confianza en grupos profesionales e instituciones.

- Percepciones ante los retos globales. 
Esta medición permite conocer el panorama comparativa en la forma que las poblaciones de estos países perciben los valores relacionados con temas claves del ámbito democrático, y político-social. En el estudio, en cada una de las dimensiones y aspectos examinados se presentan extensos datos que permiten entrar en detalle en cada uno de los aspectos examinados.

En la actualidad esta medición se lleva a cabo con una alta fiabilidad de los datos y un pequeño margen de error, y favorece la reducción de prejuicios y estereotipos que en las poblaciones de los distintos países puedan existir.

\subsection{VALORES EN LA EMPRESA}

El «Informe Randstad de valores en la empresa», recoge la opinión de más de 1.200 responsables de selección y de más de 7.800 profesionales de empresas de todos los sectores, tamaños y comunidades autónomas españolas. A través de este informe, se pueden conocer los valores preferidos por las empresas. El informe trabaja con los siguientes valores: compromiso, coraje/ energía/empuje, disciplina, empatía, generosidad, honestidad/ética/integridad, humildad/sencillez, iniciativa, lealtad/fidelidad, optimismo/positivismo, perseverancia/constancia, respeto/tolerancia, responsabilidad, serenidad, y solidaridad/superación y esfuerzo.

Los valores que buscan prioritariamente las empresas, de los diez y seis valores estudiados, son los seis que hemos señalado en cursiva. En el estudio se puede ver comparativamente los valores que demandan las empresas de los profesionales, cómo consideran realmente cómo son esos profesionales, y un ranking de los valores profesionales y empresas.

\section{LOS VALORES EN LAS ORGANIZACIONES}

Cuando se estudia el funcionamiento de las organizaciones siempre aparecen los valores juntamente con el quehacer de la institución (su misión), la proyección hacia el futuro (su visión estratégica) y los principios de actuación y sobre qué fundamentos se sostienen (los valores).

Todos los grandes autores que examinaron las organizaciones subrayaron la importancia de los mismos en la estructura y funcionamiento de las organizaciones. Kouzes y Posner (2017), autores de uno los libros más vendidos sobre el liderazgo, para señalar la importancia que tienen en el líder traen a colación una reflexión de una directora de una gran empresa cuando señala: 
Tuve que preguntarme en que creía, qué era importante para mí, qué tipo de planteamientos iba a seguir, qué quería comunicar y cuáles eran mis expectativas. En primer lugar, necesitaba saberlo y creerlo en mi interior. Me vinieron a la cabeza muchas cosas a la vez, pero tenía que centrarme en los valores fundamentales que quería representar (p. 49).

Los citados autores recomiendan a los líderes dos aspectos que deben tener en cuenta y ponerlo en práctica:

- Encuentra tu propia voz

- Afirma los valores compartidos

\subsection{LA MEDICIÓN DE LOS VALORES EN LAS ORGANIZACIONES}

Uno de los autores que ha investigado el mundo de los valores en el ámbito de las organizaciones es Brian Hall (2000) refiriéndose a las organizaciones afirma: «las organizaciones son culturas de relaciones que incrementan u obstaculizan la gestión del conocimiento. El vínculo que da cohesión a estas relaciones son las prioridades de valores de acuerdo con las que vivimos. Paradójicamente nuestras diferencias de valores son las que estimulan la creatividad y la comprensión. Sin embargo, si no compartimos unas mínimas prioridades de valores, nuestra relación no será sostenible. Lo que se requiere, pues, es mantener estas dos dimensiones en constante tensión» (p. 30).

Brian Hall subraya dos dificultades en torno a los valores:

En muchos casos, el primer problema es que no conocemos nuestros valores, que en muchos casos no son conscientes, ni siquiera para la propia persona y mucho menos a nivel grupal. De ahí se deriva la necesidad de medir los valores para conocerlos en primer lugar nosotros mismos. Una vez conocidos estos valores, podemos compartirlos con otras personas, en un grupo, en la familia; y tomar decisiones sobre lo que queremos hacer.

El segundo problema es que el conocimiento es siempre contextual y fluye, como dice Hall de un conjunto de relaciones que están sustentadas por un mínimo de prioridades de valores compartidos. Y de ahí se deriva la segunda dificultad. Como señala Nonaka y Takeuchi el conocimiento es «tácito» y «explícito», el conocimiento tácito es subjetivo y conocido por el individuo, puede incluso ser inconsciente. El conocimiento explícito está codificado, y como conocimiento explícito suele estar escrito.

La medición de valores consolida nuestro aprendizaje:

- Poniendo de manifiesto nuestras prioridades de valores, de manera que puedan ser compartidas con otras personas.

- Al compartir valores con otras personas (miembros del equipo, compañeros, amigos...), convertimos lo que era tácito en explícito. Esto hace 
posible ver lo diferentes que somos, de forma que la relación pueda comprenderse mejor.

Fredy Kofman (2001, p. 188) ante la pregunta: ¿cuáles son los valores fundamentales de las personas? Tiene una respuesta clara, se reducen a cinco: felicidad, plenitud, libertad, paz y amor. El autor basa su consideración de que nuestra estructura profunda de nuestra psiquis trasciende las diferencias superficiales que existen entre distintas culturas. Y señala como símil, que, igual que la mayoría de las personas tienen dos ojos, nariz, boca y diez dedos en las manos, también la mayoría aspiran a ser felices, en plenitud, libres, en un marco de paz y amor.

Kofman sugiere que las personas debemos hacernos preguntas,

[...] por ejemplo, si usted quisiera tener algo, por ejemplo, un coche, tiempo libre, un aumento de sueldo, o cualquier otra cosa, hágase la siguiente pregunta: "¿qué obtendría si pudiera conseguir eso?, ¿qué es para mí aún más valioso que eso en sí mismo?» Por ejemplo, la persona tiene el deseo de escribir un libro. La pregunta sería: «¿qué obtendría si pudiera conseguir eso?, ¿qué es para mí aún más valioso que eso en sí mismo?» Una respuesta podría ser: «una forma de comunicar mis ideas a gente con la que no tengo interacción directa» (puede haber más de una razón, pero para el ejemplo basta una). A continuación, tome esa respuesta, y hágase nuevamente la pregunta: "¿qué obtendría si pudiera conseguir eso (comunicar mis ideas...), ¿qué es aún más valioso para mí que eso en sí mismo?» Por ejemplo, mi segunda respuesta podría ser: «contribuir al crecimiento de gente que ni siquiera conozco». Tome de nuevo esta respuesta y pregúntese de nuevo la misma pregunta, y repita el procedimiento hasta que no pueda imaginarse una razón ulterior para querer lo que quiere. En el ejemplo, podría ser: «sentirme valioso, sentir que mi vida es importante, satisfacción, felicidad.» Con toda seguridad, afirma el autor, la respuesta será algo equivalente a la plenitud de su existencia, libertad, felicidad, paz y amor. (p. 189)

Posteriormente, analiza los cinco valores que considera más relevantes; felicidad, plenitud, libertad, paz y amor.

\subsection{El modelo de Brian Hall y Benjamin Tonna}

Entre las metodologías de estudio de los valores en las organizaciones destaca el enfoque propuesto por Hall y Tonna que ha sido aplicado en numerosos países. Una de las mayores aportaciones del modelo de Hall-Tonna es su aplicación para el conocimiento de las organizaciones y el examen de su evolución institucional. Como señala Hall (2000, p. 30): «las organizaciones son culturas de relaciones que incrementan u obstaculizan la gestión del 
conocimiento. El vínculo que da cohesión a estas relaciones son las prioridades de valores de acuerdo con las que vivimos».

La contribución de Hall y Tonna en el ámbito de la medición de valores en las organizaciones es de un gran valor y utilidad, pues a través de esta medida se puede entender mejor la vida institucional y poder mejorar su dinámica. Hall parte de la premisa que nuestro avance en el conocimiento se da por etapas, y describe la cultura corporativa y el conocimiento de los valores en cuatro niveles, tal como se refleja en la figura siguiente:

Figura 3. LA CULTURA ORganizativa, EL CONOCimiENTO y LOS VALORES

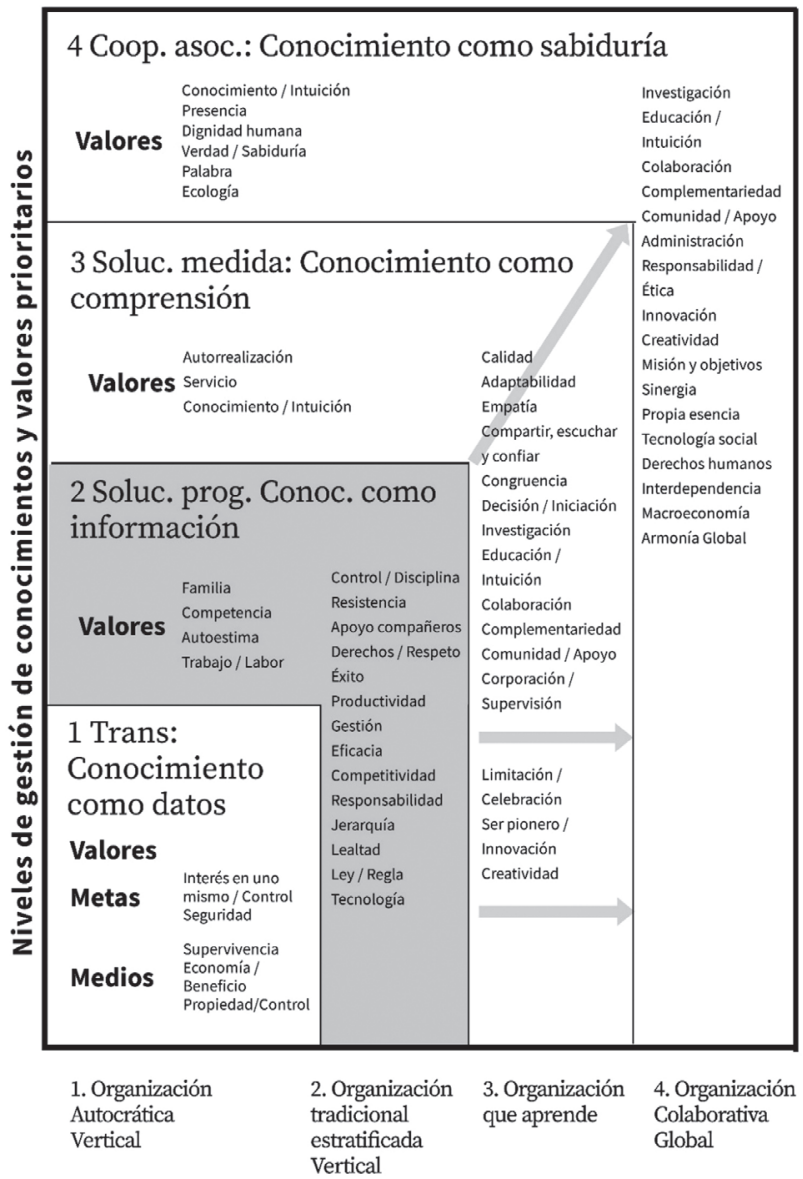

Fuente: Reproducción Hall (2000, p. 32). 
Hall distingue cuatro niveles que pueden distinguirse en el funcionamiento de las organizaciones y que pueden determinarse sus principales características. Estos niveles son:

Primer nivel: es el conocimiento como datos. Los valores son muy limitados y reflejan una visión del mundo relacionada con la seguridad y el control.

Segundo nivel: es el del conocimiento como información. Esta contiene datos agrupados, significativos en un contexto dado. Esta visión del mundo tiene que ver con la pertenencia y el mantenimiento del status quo. La razón y la conformidad domina esta etapa.

Tercer nivel: es el del conocimiento como comprensión. Este nivel supone un paso importante un cambio en las relaciones interpersonales, que conducen a la percepción intelectual personal. El conocimiento a este nivel requiere una conciencia y un nivel de valores personales relacionados con la autoridad personal, como en la iniciativa en la decisión.

Cuarto nivel: es el conocimiento como sabiduría. En este nivel, el conocimiento es relacional, pero a nivel global. En este nivel se añade la sabiduría práctica en la distribución y utilización del conocimiento para crear armonía global y aumentar la calidad de vida humana.

En la figura pueden verse los valores propios correspondientes a cada nivel. En la base de la figura, Hall sitúa la evolución de las organizaciones en cuatro estadios: Organización autocrática vertical, Organización tradicional estratificada vertical, Organización que aprende, y lo que Hall determina como Organización Colaborativa Global.

Hall considera que la medición de valores que pueda examinar de modo objetivo las discrepancias de una organización ofrecerá una ventaja competitiva a las personas lo suficientemente sabias como para utilizarla.

La medición de valores es sencillamente un instrumento esencial en los negocios globales, particularmente cuando analizamos fusiones, adquisiciones y una base compleja de clientes.

Cuando Brian Hall y Benjamin Tonna vinieron a la Universidad de Deusto para formar a un grupo de profesores, que posteriormente extendieron este enfoque y aportaron su conocimiento y desarrollo a través de distintas publicaciones. La metodología de Hall-Tonna permite el análisis cualitativo de documentos y de este modo se llevaron a cabo diferentes estudios. Los valores de la Universidad de Deusto a través de sus documentos programáticos, el estudio de los valores de la LOGSE (Bunes, Calzón, Elexpuru, Fañanás, Muñoz-Repiso y Valle, 1993), el desarrollo de los valores en las instituciones educativas (centros de enseñanza no universitarios) Eléxpuru y Medrano (2002). 


\section{LAS APORTACIONES METODOLÓGICAS SOBRE VALORES}

\subsection{La aportación de Milton Rokeach}

Milton Rokeach fue un pionero en la medición de valores y son muchos los autores deudores de sus aportaciones (Inglehart, y muy especialmente Schwartz).

Como señala Pérez Martínez (2011): Milton Rokeach (1973), considerado como el pionero de los estudios sistemáticos sobre valores desde la perspectiva de la psicología social y organizacional, también manifestó su preocupación por la carencia de consenso en cuanto al concepto científico de valor al señalar que: «el concepto de valor, más que cualquier otro, debería ocupar un lugar central y común en las distintas ciencias sociales» (p. 3). Y define el concepto de valor como: "una creencia perdurable de que un modo de conducta específico o fin en la vida, es personal o socialmente preferible sobre otro modo de conducta o fin de existencia» (p. 5).

Rokeach (1973) distingue en sus modelos distintos dos tipos de valores: terminales e instrumentales, que posteriormente Schwartz los adaptaría en sus estudios transculturales aplicando su sistema en un grupo números de países de distintos continentes. Presentamos un cuadro comparativo entre los valores señalados por ambos autores.

Tabla 1. Comparación de los valores de RoKeach y Schwartz

\begin{tabular}{|l|l|l|l|}
\hline $\begin{array}{c}\text { Valores } \\
\text { terminales según } \\
\text { Rokeach }\end{array}$ & $\begin{array}{c}\text { Valores } \\
\text { Instrumentales } \\
\text { según Rokeach }\end{array}$ & $\begin{array}{c}\text { Valores } \\
\text { terminales según } \\
\text { Schwartz }\end{array}$ & $\begin{array}{c}\text { Valores } \\
\text { Instrumentales } \\
\text { según Schwartz }\end{array}$ \\
\hline $\begin{array}{l}\text { Un mundo en } \\
\text { paz (sin guerras } \\
\text { ni conflictos) }\end{array}$ & $\begin{array}{l}\text { Ambición (trabajo, } \\
\text { esforzado, } \\
\text { aspiraciones) }\end{array}$ & $\begin{array}{l}\text { Un mundo en paz } \\
\text { (libre de guerras } \\
\text { y conflictos) }\end{array}$ & $\begin{array}{l}\text { Ambicioso } \\
\text { (trabajo duro, con } \\
\text { altas aspiraciones) }\end{array}$ \\
\hline $\begin{array}{l}\text { Seguridad familiar } \\
\text { (hacerse cargo de } \\
\text { los seres queridos) }\end{array}$ & $\begin{array}{l}\text { Mente Abierta } \\
\text { (de mentalidad } \\
\text { amplia) }\end{array}$ & $\begin{array}{l}\text { Seguridad familiar } \\
\text { (seguridad para los } \\
\text { seres queridos) }\end{array}$ & $\begin{array}{l}\text { Abierto (tolerante } \\
\text { con diferentes } \\
\text { ideas y creencias) }\end{array}$ \\
\hline $\begin{array}{l}\text { Libertad } \\
\text { (independencia, } \\
\text { libre elección) }\end{array}$ & $\begin{array}{l}\text { Capaz } \\
\text { (competente, } \\
\text { eficaz) }\end{array}$ & $\begin{array}{l}\text { Libertad (libertad } \\
\text { de acción y } \\
\text { pensamiento) }\end{array}$ & $\begin{array}{l}\text { Capaz } \\
\text { (competente, } \\
\text { eficaz) }\end{array}$ \\
\hline $\begin{array}{l}\text { Igualdad (herman- } \\
\text { dad, igualdad de } \\
\text { oportunidades } \\
\text { para todos) }\end{array}$ & $\begin{array}{l}\text { Animado } \\
\text { (alegre, gozoso) }\end{array}$ & $\begin{array}{l}\text { Igualdad (igualdad } \\
\text { de oportunidades } \\
\text { para todos) }\end{array}$ & \\
\hline
\end{tabular}




\begin{tabular}{|c|c|c|c|}
\hline $\begin{array}{c}\text { Valores } \\
\text { terminales según } \\
\text { Rokeach }\end{array}$ & $\begin{array}{l}\text { Valores } \\
\text { Instrumentales } \\
\text { según Rokeach }\end{array}$ & $\begin{array}{c}\text { Valores } \\
\text { terminales según } \\
\text { Schwartz }\end{array}$ & $\begin{array}{c}\text { Valores } \\
\text { Instrumentales } \\
\text { según Schwartz }\end{array}$ \\
\hline $\begin{array}{l}\text { Respeto por } \\
\text { uno mismo } \\
\text { (autoestima) }\end{array}$ & $\begin{array}{l}\text { Limpio (pulcro, } \\
\text { esmerado) }\end{array}$ & $\begin{array}{l}\text { Auto-Respeto } \\
\text { (creer en mi } \\
\text { propia valía). }\end{array}$ & $\begin{array}{l}\text { Limpio (aseado, } \\
\text { ordenado) }\end{array}$ \\
\hline $\begin{array}{l}\text { Felicidad (estar } \\
\text { contento) }\end{array}$ & $\begin{array}{l}\text { Valiente (defender } \\
\text { las propias } \\
\text { convicciones) }\end{array}$ & & \\
\hline $\begin{array}{l}\text { Sabiduría (una } \\
\text { comprensión } \\
\text { madura de la vida) }\end{array}$ & $\begin{array}{l}\text { Perdón } \\
\text { (disposición } \\
\text { a perdonar a } \\
\text { los demás) }\end{array}$ & $\begin{array}{l}\text { Sabiduría } \\
\text { (comprensión } \\
\text { madura de } \\
\text { la vida). }\end{array}$ & $\begin{array}{l}\text { No rencoroso } \\
\text { (dispuesto a } \\
\text { perdonar a } \\
\text { los demás) }\end{array}$ \\
\hline $\begin{array}{l}\text { Seguridad nacional } \\
\text { (protección } \\
\text { contra ataques) }\end{array}$ & $\begin{array}{l}\text { Servicial (trabajar } \\
\text { por el bienestar } \\
\text { de otros) }\end{array}$ & $\begin{array}{l}\text { Seguridad } \\
\text { Nacional (proteger } \\
\text { mi nación del } \\
\text { enemigo). }\end{array}$ & $\begin{array}{l}\text { Ayudo (trabajo } \\
\text { por los demás) }\end{array}$ \\
\hline $\begin{array}{l}\text { Salvación (salvado, } \\
\text { vida eterna) }\end{array}$ & $\begin{array}{l}\text { Honesto (sincero, } \\
\text { confiable) }\end{array}$ & $\begin{array}{l}\text { Esperanza (de } \\
\text { otra vida) }\end{array}$ & $\begin{array}{l}\text { Honesto (sincero, } \\
\text { genuino) }\end{array}$ \\
\hline $\begin{array}{l}\text { Amistad verdadera } \\
\text { (compañía cercana) }\end{array}$ & $\begin{array}{l}\text { Imaginativo } \\
\text { (audaz, creativo) }\end{array}$ & $\begin{array}{l}\text { Amistad } \\
\text { Verdadera (amigos } \\
\text { cercanos que } \\
\text { me apoyen). }\end{array}$ & $\begin{array}{l}\text { Leal (fiel a } \\
\text { mis amigos y } \\
\text { a mi grupo) }\end{array}$ \\
\hline $\begin{array}{l}\text { Sentimiento de } \\
\text { logro (contribución } \\
\text { duradera) }\end{array}$ & $\begin{array}{l}\text { Independiente } \\
\text { (confiado, } \\
\text { autosuficiente) }\end{array}$ & & $\begin{array}{l}\text { Independiente } \\
\text { (confiado, } \\
\text { autosuficiente }\end{array}$ \\
\hline $\begin{array}{l}\text { Armonía Interior } \\
\text { (sin conflictos } \\
\text { internos) }\end{array}$ & $\begin{array}{l}\text { Intelectual } \\
\text { (inteligente, } \\
\text { reflexivo) }\end{array}$ & $\begin{array}{l}\text { Armonía Interna } \\
\text { (en paz conmigo } \\
\text { mismo). }\end{array}$ & $\begin{array}{l}\text { Moderado } \\
\text { (evitando los } \\
\text { extremos en } \\
\text { sentimientos } \\
\text { y acciones) }\end{array}$ \\
\hline $\begin{array}{l}\text { Una vida cómoda } \\
\text { (una vida próspera) }\end{array}$ & $\begin{array}{l}\text { Lógico } \\
\text { (congruente, } \\
\text { racional) }\end{array}$ & & \\
\hline $\begin{array}{l}\text { Amor maduro } \\
\text { (intimidad sexual } \\
\text { y espiritual) }\end{array}$ & $\begin{array}{l}\text { Cariñoso } \\
\text { (afectuoso, tierno) }\end{array}$ & $\begin{array}{l}\text { Amor Maduro } \\
\text { (relación profunda } \\
\text { de intimidad } \\
\text { emocional } \\
\text { espiritual). }\end{array}$ & $\begin{array}{l}\text { Disfruto de la } \\
\text { vida (comida, } \\
\text { sexo, ocio) }\end{array}$ \\
\hline
\end{tabular}




\begin{tabular}{|c|c|c|c|}
\hline $\begin{array}{c}\text { Valores } \\
\text { terminales según } \\
\text { Rokeach }\end{array}$ & $\begin{array}{c}\text { Valores } \\
\text { Instrumentales } \\
\text { según Rokeach }\end{array}$ & $\begin{array}{l}\text { Valores } \\
\text { terminales según } \\
\text { Schwartz }\end{array}$ & $\begin{array}{l}\text { Valores } \\
\text { Instrumentales } \\
\text { según Schwartz }\end{array}$ \\
\hline $\begin{array}{l}\text { Un mundo de } \\
\text { belleza (de la } \\
\text { naturaleza y } \\
\text { de las artes) }\end{array}$ & $\begin{array}{l}\text { Obediente } \\
\text { (diferente, } \\
\text { respetuoso) }\end{array}$ & $\begin{array}{l}\text { Un Mundo de } \\
\text { Belleza (belleza } \\
\text { en la naturaleza } \\
\text { y en las artes). }\end{array}$ & \\
\hline $\begin{array}{l}\text { Placeres (una } \\
\text { vida gozosa y } \\
\text { despreocupada) }\end{array}$ & $\begin{array}{l}\text { Cortés (amable, } \\
\text { formal) }\end{array}$ & $\begin{array}{l}\text { Placer } \\
\text { (satisfacción } \\
\text { de deseos). }\end{array}$ & $\begin{array}{l}\text { Indulgente } \\
\text { consigo mismo } \\
\text { (permitiéndome } \\
\text { placeres) }\end{array}$ \\
\hline $\begin{array}{l}\text { Reconocimiento } \\
\text { social ( respeto, } \\
\text { admiración) }\end{array}$ & $\begin{array}{l}\text { Responsable } \\
\text { (fiable, confiable) }\end{array}$ & $\begin{array}{l}\text { Reconocimiento } \\
\text { Social (respeto, } \\
\text { aprobación de } \\
\text { los demás }\end{array}$ & $\begin{array}{l}\text { Logro de éxitos } \\
\text { (consiguiendo } \\
\text { metas) }\end{array}$ \\
\hline $\begin{array}{l}\text { Una vida } \\
\text { emocionante (una } \\
\text { vida estimulante, } \\
\text { activa) }\end{array}$ & $\begin{array}{l}\text { Controlado } \\
\text { (limitado, auto } \\
\text { disciplinado) }\end{array}$ & $\begin{array}{l}\text { Una Vida } \\
\text { Excitante } \\
\text { (experiencias } \\
\text { estimulantes). }\end{array}$ & $\begin{array}{l}\text { Atrevido (busco } \\
\text { aventuras y } \\
\text { riesgos) }\end{array}$ \\
\hline
\end{tabular}

Fuente: elaboración propia.

Schwartz además de estos valores comparativos añade otros valores:

Valores terminales:

Poder social (control sobre otros, dominio), trabajo (ganarse la vida duramente), sentimiento de pertenencia (sentimiento que otros se preocupan de mí), orden social (estabilidad de la sociedad),buenos modales (cortesía, buenas maneras), riqueza (posesiones materiales), reciprocidad de favores (evitar estar en deuda con los demás), creatividad (originalidad, imaginación), respeto por la tradición (mantener las costumbres conservadas a lo largo del tiempo), autodisciplina (autocontrol, resistencia a las tentaciones), distanciamiento (de las inquietudes mundanas), unión con la naturaleza (integrarse con la naturaleza), una vida variada (llena de desafíos, novedad y cambio), intimidad (búsqueda de los propios sentimientos y vivencias), autoridad (el derecho a liderar o mandar), un mundo de belleza (belleza en la naturaleza y en las artes), justicia social (corregir injusticias, preocuparse por los débiles), privacidad (derecho a tener un ámbito personal).

y otros valores instrumentales: humilde (modesto, pasa desapercibido), atrevido (busco aventuras y riesgos), honro a los padres y mayores (mostrándoles respeto), sano (no enfermo física o mentalmente), acepto mi parte 
en la vida (sometiéndome a mis circunstancias), cuido mi imagen pública (protegiendo mi «imagen»), creyente (manteniendo creencias y fe religiosa), Responsable (digno de confianza, fiable), curioso (interesado por todo, indagador).

\subsection{Características de los valores Según Shalom Schwartz}

Después de una revisión de varios modelos sobre valores (Rokeach, Inglehart, Bilsky, Allport, Vernon y Lindzey entre otros), Schwartz considera que hay un acuerdo bastante generalizado en cinco rasgos comunes en la conceptualización de los valores:

1. Un valor es una creencia.

2. Que pertenece a fines deseables o a formas de comportamiento.

3. Que trasciende las situaciones específicas.

4. Que guía la selección o evaluación de comportamientos, personas y sucesos.

5. Que se ordena por su importancia relativa a otros valores para formar un sistema de prioridades de valores.

Estos son los rasgos de valores que los distinguen de otros conceptos como necesidades, actitudes. Las actitudes son las predisposiciones a reaccionar positiva o negativamente en un cierto grado o intensidad, con respecto a objetos, ideas, instituciones o personas. Por ejemplo, uno puede estar a favor o en contra de instituciones como el aborto, la ley «trans», el «teletrabajo», etc., mostrando el grado de acuerdo o desacuerdo con cada término.

Las necesidades son la carencia de las cosas que son menester para la conservación de la vida. Estas hacen referencia a las necesidades básicas señaladas por Abraham Maslow, pero existen otro tipo de necesidades. Este autor hizo famosa su pirámide de necesidades, que se sigue utilizando cuando se desea definir las necesidades humanas. El concepto de pirámide es debido que mientras no se cumplan las necesidades denominadas básicas, no pueden lograrse las superiores. Van en orden ascendente desde las necesidades más básicas hasta las más elevadas.

Sin embargo, estos rasgos de conceptualización de los valores (creencia, fines, conductas, actitudes) no nos dicen nada del contenido sustancial de los valores, de cuáles son los distintos tipos de valores que existen.

Diversos autores han estructurado los valores de diverso modo. Schwartz define los valores como metas deseables y transituacionales, que varían en 
importancia que sirven como principios en la vida de una persona o de una institución. Implícita en esta definición de valores como metas está que:

1) sirven a los intereses de alguna entidad social;

2) pueden motivar a la acción, dándole dirección e intensidad emocional;

3) funcionan como criterios para juzgar y justificar la acción; y

4) se adquieren tanto a través de la socialización en los valores del grupo dominante como a través de la experiencia personal de aprendizaje.

\subsubsection{Tipos de valores}

Según Schwartz, los valores representan, en forma de metas conscientes, las respuestas que todos los individuos y sociedades deben dar a tres requisitos universales:

1. las necesidades de los individuos en tanto que organismos biológicos;

2. los requisitos de la interacción social coordinada, y

3. los requisitos para el correcto funcionamiento y supervivencia de los grupos.

Este autor revisó diversos modelos de valores de autores de gran prestigio y señaló diez dimensiones que considera que recogen todas las posibles dimensiones que se pueden establecer a nivel intercultural de cualquier país y cultura. Estas dimensiones son las siguientes:

Aunque las diez categorías son deducidas apriorísticamente de un marco teórico, Schwartz subraya que es posible clasificar en alguna de ellas todos los ítems de las listas específicas de valores encontradas en distintas sociedades del mundo. Las diversas culturas diferirían en el peso otorgado a cada uno de los diez valores universales.

A continuación, se describen los 10 valores genéricos. Cada uno expresa un objetivo motivacional específico. La descripción puntualiza cómo se deriva cada valor de los requerimientos humanos universales y enumera ítems específicos de valor utilizados en los cuestionarios de los estudios empíricos (Schwartz, 1992, 1994, 1999 y 2006).

El autor después de una de las investigaciones de las más extensas en países, por tanto, se trata de una investigación transcultural, estableció los tipos de valores que se pueden encontrar en todas las culturas, como se reflejan en la siguiente tabla: 
Tabla 2. Tipos motivacionales de VALORES

\begin{tabular}{|l|l|l|}
\hline \multicolumn{1}{|c|}{ Definición } & \multicolumn{1}{|c|}{ Ejemplos de Valores } & \multicolumn{1}{|c|}{ Fuentes } \\
\hline $\begin{array}{l}\text { Poder: estatus social sobre las } \\
\text { personas y los recursos. }\end{array}$ & $\begin{array}{l}\text { Poder social. Autoridad, } \\
\text { Riqueza }\end{array}$ & $\begin{array}{l}\text { Interacción. } \\
\text { Grupo }\end{array}$ \\
\hline $\begin{array}{l}\text { Logro: éxito personal mediante } \\
\text { la demostración de competencia } \\
\text { según criterios sociales. }\end{array}$ & Exitoso, Capaz Ambicioso & $\begin{array}{l}\text { Interacción. } \\
\text { Grupo }\end{array}$ \\
\hline $\begin{array}{l}\text { Hedonismo: placer y gratificación } \\
\text { sensual para uno mismo. }\end{array}$ & Placer. Disfrutar de la vida & Organismo \\
\hline $\begin{array}{l}\text { Estimulación: entusiasmo, } \\
\text { novedad, y reto en la vida. }\end{array}$ & $\begin{array}{l}\text { Atrevido. Vida variada. } \\
\text { Vida excitante }\end{array}$ & Organismo \\
\hline $\begin{array}{l}\text { Autodirección: pensamiento } \\
\text { independiente y elección de la } \\
\text { acción, creatividad, exploración. }\end{array}$ & $\begin{array}{l}\text { Creatividad. Curioso. } \\
\text { Libertad }\end{array}$ & $\begin{array}{l}\text { Organismo. } \\
\text { Interacción }\end{array}$ \\
\hline $\begin{array}{l}\text { Universalismo: comprensión, } \\
\text { aprecio, tolerancia y protección } \\
\text { del bienestar de todas las personas } \\
\text { y de la naturaleza. }\end{array}$ & $\begin{array}{l}\text { Tolerancia. Justicia Social. } \\
\text { Igualdad. Protección del } \\
\text { Medio Ambiente }\end{array}$ & $\begin{array}{l}\text { Grupo. } \\
\text { Organismo }\end{array}$ \\
\hline $\begin{array}{l}\text { Benevolencia: preservación e } \\
\text { intensificación del bienestar de las } \\
\text { personas con las que uno está en } \\
\text { contacto personal frecuente. }\end{array}$ & $\begin{array}{l}\text { Ayuda Honesto. } \\
\text { No rencoroso }\end{array}$ & $\begin{array}{l}\text { Interacción. } \\
\text { Grupo }\end{array}$ \\
\hline $\begin{array}{l}\text { Tradición: respeto, compromiso } \\
\text { y aceptación de las costumbres e } \\
\text { ideas que proporciona la cultura } \\
\text { tradicional o la religión. }\end{array}$ & $\begin{array}{l}\text { Humilde. Devoto. } \\
\text { Aceptar mi parte en la Vida } \\
\text { Grupo }\end{array}$ \\
\hline $\begin{array}{l}\text { Conformón. } \\
\text { acciones, inclinaciones e impulsos } \\
\text { que pudiesen molestar o herir } \\
\text { a otros y violar expectativas o } \\
\text { normas sociales. }\end{array}$ & $\begin{array}{l}\text { Buenos modales. Obediente. } \\
\text { Honra a los Padres y a los }\end{array}$ & $\begin{array}{l}\text { Grupores } \\
\text { Grupo }\end{array}$ \\
\hline $\begin{array}{l}\text { Seguridad: seguridad, armonín. y } \\
\text { estabilidad de la sociedad, de las } \\
\text { relaciones y de sí mismo. }\end{array}$ & $\begin{array}{l}\text { Seguridad Nacional. } \\
\text { Orden Social. Limpio }\end{array}$ \\
\hline
\end{tabular}

Fuente: reproducción Schwartz (2001, p. 56) ${ }^{2}$

2 Se especifican las tres fuentes y su significado. Organismo= Necesidades universales de los individuos como organismos biológicos. Interacción= Requisitos 
De estos tres requisitos universales se derivan diez tipos motivacionales de valores, tal como se exponen en la tabla, cada uno con la definición de su meta central, y algunos ejemplos de valores. El propio Schwartz señala que los valores que representan la meta de encontrar sentido a la vida (por ejemplo, sentido en la vida, una vida espiritual, armonía interna) cumplen los requisitos definicionales para ser clasificados como el undécimo tipo de valores, aunque puede no ser reconocido implícitamente entre las culturas estudiadas.

\subsubsection{Estructura de relaciones entre los valores}

Los contenidos anteriores de los valores no señalan su contenido sustantivo. Por ello, Schwartz desarrolló una tipología de los distintos tipos de valores basándose en el principio en que los individuos para adaptarse al contexto social, los individuos transforman las necesidades inherentes a la existencia humana y la expresan en el lenguaje de los valores específicos de modo que puedan comunicarse. Los valores representan en forma de metas deseables, las respuestas que todos los individuos y sociedades deben dar a los tres requisitos universales citados anteriormente.

Varios investigadores han derivado empíricamente tipologías del contenido de valores. Sin embargo, no han seguido las intuiciones de Rokeach de que al menos algunos tipos de valores deben ser interdependientes porque se encuentran en oposición entre sí (moral versus competencia, personal versus social).

El patrón total de relaciones de conflicto y compatibilidad entre las prioridades de valores que se postula que estructuran el sistema de valores está representado en la figura 3 .

Los tipos de valor en competición emanan en direcciones opuestas del centro; los tipos compatibles se encuentran próximos a lo largo del círculo. La situación de tradición fuera de conformidad, implica según el autor, que estos dos tipos de valores comparten una misma meta motivacional, la subordinación del yo a favor de expectativas sociales impuestas.

universales para la coordinación de la interacción social. Y Grupo= Emerge cuando las personas entran en contacto con los que están fuera de su grupo primario extenso, reconocen la interdependencia intergrupal, y se dan cuenta de la escasez de los recursos naturales. 
Figura 3. Modelo teórico ENTE Los tiPos MOtIVACIONALES, TIPOS DE VALORES DE ORDEN SUPERIOR, Y DIMENSIONES DE VALORES BIPOLARES (SCHWARTZ, 1992)

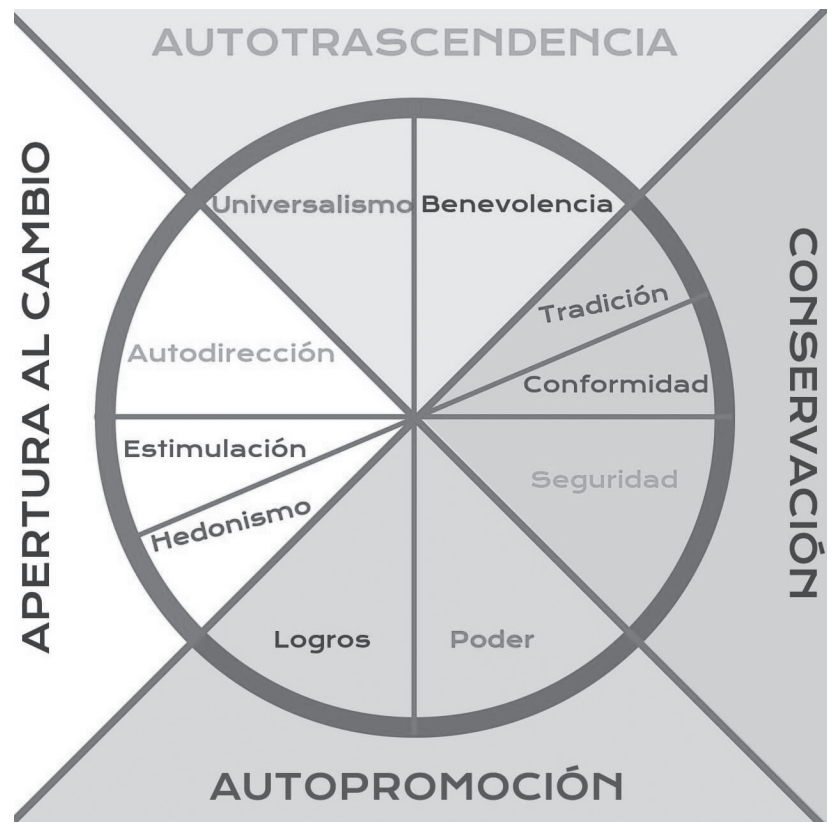

Fuente: reproducción Schwartz (2001, p. 59).

El círculo refleja los análisis de los conflictos y las compatibilidades, que probablemente puedan suceder con la realización de otros tipos de valores. Por ejemplo, el llevar a cabo los valores de logro pueden entrar en conflicto con los valores de benevolencia: la búsqueda del éxito personal es posible que obstruya las acciones dirigidas a mejorar el bienestar de los demás que necesitan nuestra ayuda. De forma similar, la búsqueda de valores tradicionales entra en conflicto con seguir los valores de estimulación: aceptar las costumbres culturales y religiosas y las ideas transmitidas del pasado es posible que inhiba la novedad, el desafío y el entusiasmo. Por otra parte, el seguir los valores de benevolencia y de conformidad resulta compatible, pues ambos implican comportarse de una forma aprobada por nuestro grupo íntimo. 
Figura 4. LOS ÉNFASIS COMPARTIDOS

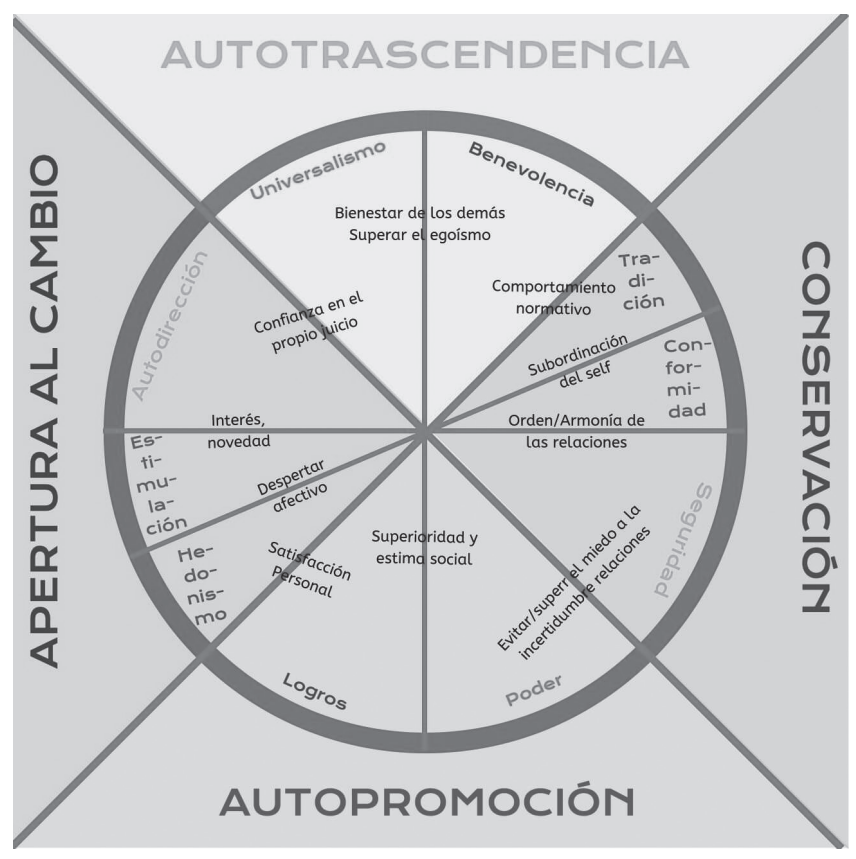

Fuente: elaboración propia.

a. Poder y logro, ambos enfatizan la superioridad y la estima social.

b. Logro y hedonismo, ambos enfatizan en la satisfacción personal.

c. Hedonismo y estimulación, ambos implican el deseo del despertar afectivo.

d. Estimulación y autodirección, ambos comparten el interés intrínseco por la novedad y la maestría.

e. Autodirección y universalismo, ambos expresan confianza en el propio juicio y confort con la diversidad de la existencia.

f. Universalismo y benevolencia, ambos se ocupan del bienestar de los demás y de trascender los intereses egoístas.

g. Benevolencia y conformidad, ambos demandan el comportamiento normativo que promueve las relaciones cercanas.

h. Benevolencia y tradición, ambos promueven la devoción al propio endogrupo. 
i. Conformidad y tradición, ambos implican subordinación del self a favor de expectativas sociales impuestas.

j. Tradición y seguridad, ambos destacan el preservar los acuerdos sociales existentes para dar seguridad a la vida.

k. Conformidad y seguridad, ambos enfatizan proteger el orden y la armonía en las relaciones.

1. Seguridad y poder, ambos subrayan el evitar o superar el miedo a la incertidumbre mediante el control de las relaciones y de los recursos.

La metodología planteada por Schwartz es quizás la más comprobada a nivel empírico y comprobada en muy diferentes países, por eso adquiere un valor transcultural que permite análisis comparativos.

Como ha quedado claro en el cuadro comparativo expuesto anteriormente, el modelo de Schwartz se fundamenta claramente en Rokeach, ampliado en una serie de valores después de una extensa investigación y con muchos estudios de contraste realizados por otros investigadores. Actualmente, es la metodología más precisa para medir los valores transculturales en las diferentes culturas desde la perspectiva universal.

\section{CONCLUSIONES}

1. Queda patente, la relevancia de los valores y su extensión en todos los ámbitos de la vida humana (personal, familiar, educativo, social, institucional y universal). Los valores ayudan a explicar cualquiera de estos ámbitos: su situación y su evolución.

2. Las diferentes metodologías aportan matices y pertinencia en función del área o foco de medición de los valores, encontrando enfoques más adecuados según el interés del objeto a evaluar. Metodologías para medir y desarrollar valores en el ámbito educativo, para evaluar los valores individuales desde una perspectiva psicológica-social, instrumentos para comprender y determinar los valores y su efecto dentro de las organizaciones. Poder obtener una fotografía valórica del mundo, lo que hace relativamente poco parecería un sueño inalcanzable.

3. Posiblemente, será difícil encontrar un término como el de valor, que permita conocer con profundidad la intimidad de las personas, de las instituciones y de la propia sociedad en general. Porque los valores reflejan la visión, el deseo, el comportamiento, la motivación, la personalidad, y todo ello, desde un concepto como el de los valores. Se trata de un constructo muy poderoso para comprender la vida humana en su sentido más profundo. 
4. Las instituciones públicas y privadas saben bien el potencial que tienen los valores, por ende, todas ellas elaboran y hacen explícito los mismos en sus documentos programáticos, en sus principios operativos y en sus mensajes internos y externo a los diferentes destinatarios.

5. Finalmente, los valores ayudan a entender y explicar mejor la motivación humana, y la propia identidad como personas en un mundo cambiante. Y estos valores y contravalores se reflejan en la educación, en el arte, en la política y se pueden examinar a lo largo de la Historia, como se ha reflejado en todas las épocas por autores contemporáneos.

\section{REFERENCIAS}

Agúndez Agúndez, M. (2008). El paradigma universitario Ledesma-Kolvenbach. Revista de Fomento Social, 252(63), 603-631.

Agúndez Agúndez, M. (2008b) (Ed.). Discursos universitarios. Peter-Hans Kolvenbach. Madrid: UNIJES.

Alarcos, F. J. y García, J. (2009). Valores. En Francisco José Alarcos (Dir). 10 palabras clave en la construcción personal. Pamplona: Editorial Verbo Divino.

Aramburuzabala, P., Cerrillo, R. y Tello, I. (2015). Aprendizaje-Servicio: Una propuesta metodológica para la introducción de la sostenibilidad curricular en la universidad. Profesorado. Revista del Currículum y Formación del Profesorado, 19(1), 79-85.

Bautista Vallejo, J. M. (2001). Actitudes y Valores. XXI, Revista de Educación, 3, 189196.

Camps, V. (1998). El valor del Civismo. Cuadernos monográficos del ICE. Bilbao: Universidad de Deusto.

Elexpuru, I. y Medrano, C. (2001). Desarrollo de los valores en las Instituciones Educativas. Bilbao: Mensajero.

Elzo, J. (1994). Jóvenes españoles 94. Madrid: Fundación Santa María.

Elzo, J. (1996). Los valores en la sociedad vasca: su evolución en los años 1990-1995. Vitoria: Servicio Publicaciones del Gobierno Vasco.

Elzo, J. (1999) (Coord.). Jóvenes españoles 99. Madrid: Fundación Santa María.

Elzo. J. (2002). Los valores de los vascos y navarros ante el nuevo milenio: tercera aplicación de la encuesta europea de valores (1990, 1995, 1999). Bilbao: Universidad de Deusto.

García, A. (2018). Service Learning and Responsible University Social Innovation in the Project Hiri Lagunkoiak-Ciudades Amigables-Friendly Cities 4All. (Tesis doctoral). Bilbao: Universidad de Deusto.

García, A., Mugarra, A. y Villa, A. (2016). Innovación Social Universitaria como marco para la fundamentación, desarrollo y evaluación comunitaria de proyectos de Aprendizaje-Servicio. Educación y Diversidad. Revista Inter-universitaria de Investigación sobre Discapacidad e Interculturalidad, 10(2), 77-86. 
García, A., Mugarra, M. y Villa, A. (2016). Responsible University Social Innovation as a framework for the theoretical foundation, development and evaluation in community of Service-Learning projects. Education and diversity, 10(2),77-86.

Gil Martínez, R. (1998). Diez valores en el aula y la tutoría: una experiencia. Cuadernos monográficos del ICE. Bilbao: Universidad de Deusto.

González, P. (2006). Jóvenes españoles 2005. Madrid: Fundación Santa María.

Hall, B. (2000). El desarrollo de valores y las organizaciones que aprenden. En A. Villa (Coord.). Liderazgo y organizaciones que aprenden (pp. 27-53). Bilbao: ICE de la Universidad de Deusto.

Hirsch, A. (1998), México: valores nacionales. Visión panorámica sobre las investigaciones de valores nacionales. México: Gernika.

Howe, L. y Howe, M. (1977). Cómo personalizar la educación. Perspectivas de la clarificación de valores. Madrid: Santillana.

Inglehart, R. (1977). The silent revolution: Changing values and political styles among Western Publics. Princeton, NJ: Princeton University Press.

Inglehart, R. (1984). El Post-Materialismo en un entorno de inseguridad. En J.I. Ruiz Olabuénaga (Ed.) Estilos de Vida e Investigación Social (pp. 171-218). Bilbao: Mensajero.

Inglehart, R. (2005). Modernización y cambio cultural: la persistencia de los valores tradicionales. Quaderns de la Mediterránia, 5, 21-32.

Inglehart, R. y Welzel, Ch. «The WVS Cultural Map of the World». WVS. Archived from the original on October 19, 2013. Recuperado 6 febrero 2021.

Inglehart-Welzel Culture Map - Business Insider. Business Insider. 3 July 2014. Retrieved 6 October 2014.

Kofman, F. (2001). La nueva con-ciencia de los negocios. En F. Koffman (Ed) Metamanagement. Capítulo 24 Valores y Virtudes. Tomo 3, 183-238. Barcelona: Granica.

Kolvenbach, P. H. (1985/2008). La universidad jesuítica hoy. Sobre el ministerio de la enseñanza. En Agúndez Agúndez, M. (ed.). Kolvenbach, P. H. Discursos universitarios (pp. 31-43). Madrid: UNIJES.

Kolvenbach, P. H. (1989/2008). Características de nuestra educación. En Agúndez Agúndez, M. (ed.). Kolvenbach, P. H. Discursos universitarios (pp. 55-73). Madrid: UNIJES.

Kolvenbach, P. H. (1989/2008). En el segundo centenario de la enseñanza jesuítica en los Estados Unidos. En Agúndez Agúndez, M. (ed.). Kolvenbach, P. H. Discursos universitarios (pp. 74-86). Madrid: UNIJES.

Kolvenbach, P. H. (1990/2008). Un nuevo modelo de universidad. En Agúndez Agúndez, M. (ed.). Kolvenbach, P. H. Discursos universitarios (pp. 87-98). Madrid: UNIJES.

Kolvenbach, P. H. (1991/2008). Centenario universidad Pontificia Comillas. En Agúndez Agúndez, M. (ed.). Kolvenbach, P. H. Discursos universitarios (pp. 101-112). Madrid: UNIJES.

Kolvenbach, P. H. (1992/2008). Centenario universidad Pontificia Universidad Católica y Evangelización de la Cultura. En Agúndez Agúndez, M. (ed.). Kolvenbach, P. H. Discursos universitarios (pp. 113-123). Madrid: UNIJES. 
Kolvenbach, P. H. (2001/2008). La universidad de la Compañía de Jesús a la luz del Carisma Ignaciano. En Agúndez Agúndez, M. (ed.). Kolvenbach, P. H. Discursos universitarios (pp. 113-123). Madrid: UNIJES.

Kouzes, J. M. y Posner, B. Z. (2018). El desafío del Liderazgo. Barcelona: Editorial Reverté.

Küng, H. (2012). La crisis económica global hace necesaria una ética global. El manifiesto por una ética económica global. En BBVA (Ed), Valores y Ética para el siglo XXI (pp. 29-46). Madrid: Open Mind-BBVA.

López-Yarto, L. y Morales, P. (1985). Jerarquía de valores en la educación española: estudio comparativo de dos tipos de población escolar. Miscelánea Comillas, 43, 93-153.

Maslow, A. H. (1964): Religions, values and peak-experiences. Columbus, Ohio State University Press.

Morales, P. (1995). El Influjo de los profesores y las universidades en las actitudes y valores de los alumnos. Miscelánea Comillas, 53, 487-510.

Morales, P. (2002). La evaluación de los valores y de las actitudes. En M. I. Serrano González, (Ed.), La educación para la salud en el siglo XXI (pp. 67-88). Madrid: Alezeia y Díaz de Santos.

Morales, P. (2006). Implicaciones para el profesor de una enseñanza centrada en el alumno. Miscelánea Comillas, 64(124), 11-38.

Morales, P. (2011). Evaluación de los valores: análisis de listas de ordenamiento. Universidad Comillas. Recuperado de http://www.upcomillas.es/personal/peter/otrosdocumentos/ValoresMetodo.pdf

Opazo, H., Aramburuzabala, P. y McIlrath, L. (2019). Aprendizaje-Servicio en la educación superior. Bordón, 71(3),15-23.

Oyola, E. M. (2012). Aprendizaje en Servicio para el fortalecimiento de la autoestima y desarrollo de valores. Puerto Rico: Bibliográficas.

Reiner, H. (1985). Bueno y malo. Madrid: Encuentro.

Rokeach, M. (1968). Beliefs, attitudes and values. San Francisco: Jossey-Bass.

Rokeach, M. (1973). The nature of human values. Nueva York: Free Press.

Santos Guerra, M. A. (1990). Hacer visible lo cotidiano. Madrid: Akal.

Santos Rego, M. A., Sotelino Losada, A. y Lorenzo Moledo, M. (2015). AprendizajeServicio y misión cívica de la universidad. Barcelona: Ediciones Octaedro.

Scheler, M. (1972). El saber y la cultura. Buenos Aires: La Pléyade.

Schwartz, S. H. (2001). ¿Existen aspectos universales en la estructura y contenido de los valores humanos? En M. Ros y V. Valdiney (Coords), Psicología social de los valores humanos. Desarrollos teóricos, metodológico y aplicados (pp. 53-77). Madrid: Biblioteca Nueva.

Schwartz, S. H. (2006). A Theory of Cultural Value Orientations: Explication and Applications. Comparative Sociology, 5(2-3), 137-182.

UNIJES (2007). Reflexiones $\mathrm{i}+\mathrm{m}$ ante los nuevos desafios universitarios [Documentomarco]. Recuperado de: https://unijes.net/recursos/documentos/

Villa, A. (2013). ISUR. Un modelo de evaluación de la Innovación Social Universitaria Responsable. Bilbao: Tuning. 
Villa, A. y Lemke, C. A. (2016). El Paradigma Ledesma-Kolvenbach: Origen y concreción en el ámbito universitario. ARBOR Ciencia, Pensamiento y Cultura, 192-782, a358.

Williams, R. M. (1968): Values. En E. Shills (Ed.), International Encyclopaedia of the social sciences. New York: McMillan.

Yamamoto, K. (1969). Many Faces of Teaching. En K. Yamamoto (Ed.). Teaching, Essays, Reading. Boston: Mifflin and Co. 
